Décadrages Décadrages

cinéma, à travers champs Cinéma, à travers champs

$20 \mid 2012$

Peter Watkins

\title{
Le théâtre performatif et les reportages de "politique-fiction » de Peter Watkins
}

\section{François Bovier et Hamid Taieb}

\section{(2) OpenEdition}

1 Journals

Édition électronique

URL : https://journals.openedition.org/decadrages/225

DOI : $10.4000 /$ decadrages. 225

ISSN : 2297-5977

Éditeur

Association Décadrages

Édition imprimée

Date de publication : 10 avril 2012

Pagination : 8-33

ISBN : 978-2-9700668-4-2

ISSN : 2235-7823

Référence électronique

François Bovier et Hamid Taieb, «Le théâtre performatif et les reportages de " politique-fiction » de Peter Watkins », Décadrages [En ligne], 20 | 2012, mis en ligne le 10 avril 2013, consulté le 03 avril 2022. URL : http://journals.openedition.org/decadrages/225 ; DOl : https://doi.org/10.4000/ decadrages. 225 


\section{Le théâtre performatif et les reportages}

\section{de "politique-fiction" de Peter Watkins}

\section{par François Bovier et Hamid Taieb}

1 Voir Sébastien Layerle, "Une juste appropriation des faits. The Forgotten Faces et les années Playcraft, (1956-1962)", dans Sébastien Denis et Jean-Pierre Maghit (éd.), L'insurrection médiatique. Médias, histoire et documentaire dans le cinéma de Peter Watkins, Pessac, Presses universitaires de Bordeaux, 2010, pp. 77-90; Antoine de Baecque, "Peter Watkins en direct de l'histoire", L'histoirecaméra, Paris, Gallimard, 2008, pp. 207-257.

2 Huw Wheldon, le responsable des pro grammes documentaires de la BBC, engage Peter Watkins en tant qu'assistant producteur en 1963. La BBC a déjà diffusé plusieurs courts métrages amateurs de Peter Watkins, qui ont obtenu l'Oscar des dix meilleurs films amateurs de l'année (The Diary of an Unknown Soldier, 1959; The Forgotten Faces, 1961). Au sein de la BBC, Watkins travaille notamment sur The Life and Times of Marshall Tito de Stephen Hearth, et réalise dans ce contexte son premier long métrage professionnel, Culloden (1964). Mais en 1965, face au refus de la BBC de diffuser The War Game (La bombe, 1965), Watkins quitte ses fonctions. (Voir Joseph A. Gomez, Peter Watkins, Boston, Twayne Publishers, 1979, p. 33).

3 "Dans un monde qui est bien le nôtre, celui que nous connaissons, $[. .$.$] se produit un évé-$ nement qui ne peut s'expliquer par les lois de ce même monde familier. Celui qui perçoit l'événement doit opter pour l'une des deux solutions possibles: ou bien il s'agit d'une illusion des sens, d'un produit de l'imagination et les lois du monde restent alors ce qu'elles sont; ou bien l'événement a véritablement eu lieu, il est partie intégrante de la réalité, mais alors cette réalité est régie par des lois inconnues de nous. [...] Le fantastique occupe le temps de cette incertitude; dès qu'on choisit l'une ou l'autre réponse, on quitte le fantastique pour entrer dans un genre voisin, l'étrange ou le merveilleux. " (Tzvetan Todorov, Introduction à la littérature fantastique, Paris, Seuil, 1970, p. 29).
Les films de reportage de Peter Watkins, dont le mode documentarisant est perturbé par l'introduction de structures fictionnelles qui relèvent de l'ordre de l'anticipation, mobilisent systématiquement une stratégie de choc, dans une perspective politique: il s'agit de provoquer et de prendre à partie le spectateur, dans l'espoir que celui-ci se retourne de manière critique sur le façonnage des discours et de la représentation dans les mass media. Cette stratégie, qui repose sur la systématisation de tropes télévisuels tels que l'adresse à la caméra, le tournage caméra à l'épaule et la présence insinuante d'une voix over en position de surplomb, est indissociable d'un double ancrage dans le mouvement du cinéma amateur et dans les unités de production documentaire de la télévision britannique de la fin des années 19501. En effet, les films de Watkins radicalisent les expérimentations sur la mise en scène et la direction d'acteurs qui caractérisent le cinéma amateur anglais, et poussent à outrance les procédés formels associés aux reportages sociaux de la BBC2. Le cadrage des acteurs non professionnels en gros plans ou à travers des compositions de foule, la mobilité de la prise de vue et la réactivité de la caméra tenue à l'épaule, ainsi que l'appropriation du dispositif de l'entretien filmique concourent à induire un «effet de réel». $\mathrm{Ce}$ «style» immédiatement reconnaissable est néanmoins problématisé, contredit par l'ambivalence et l'ambiguïté de l'énonciation, l'identité de l'instance de la narration filmique étant déstabilisée ou érodée par une dimension "fantastique» (se situant, d'après la définition de Todorov ${ }^{3}$, en deçà ou par-delà les genres de l'étrange et du merveilleux - ou bien plutôt, ici, de l'borrifique).

Culloden (1964), une production de la BBC, incarne exemplairement ce processus d'appropriation de codes génériques à travers une dynamique de subversion du mode de représentation documentaire. La multiplicité des points de vue mis en scène, l'ubiquité de la caméra et la mobilisation de sources historiques ont pour effet de déconstruire 
l'histoire officielle de la bataille de Culloden, telle qu'elle est enseignée dans les manuels scolaires. Les protagonistes du conflit sont d'emblée montrés sous un jour peu reluisant, à travers une série de portraits critiques commentés par une voix over sarcastique: les Anglais sont présentés comme des mercenaires sans merci et les Highlanders comme des gueux épuisés, conduits arbitrairement par un chef fat et dépourvu de toute intelligence du combat. La médiatisation de la bataille par l'intermédiaire d'un chroniqueur parachève ce mouvement de récusation de "l'histoire des vainqueurs»: incarné par Andrew Henderson, le biographe du duc de Cumberland, le point de vue privilégié et censément objectif du "reporter» est mis en cause au sein même du dispositif de focalisation du film. Dans une séquence clef du film, le chroniqueur, dont le discours est entrecoupé par la charge des canons, observe l'offensive des rebelles à travers une longue-vue, qui condense les points de vue de l'opérateur, du reporter et du stratège sur les lieux de la bataille. Abrité derrière un muret, il décrit les échanges de tirs, tandis que la caméra mime une perte de stabilité dans le feu du combat. En contrechamp, une série de plans représente confusément la déroute des lignes rebelles. Ce contrechamp se précise par la suite à travers l'intervention d'une voix over et d'images saisissantes qui explicitent les effets des tirs de boulets, représentant un jeune combattant la jambe sectionnée, un deuxième éviscéré et un troisième en état de choc. La mise en relation de la voix over, au ton neutre, proche de la description clinique, et de gros plans de jeunes hommes hurlant de douleur, suscite la sidération et l'hébètement du spectateur. La charge critique est radicale et la cruauté manifeste, comme les journalistes n’ont pas manqué de le souligner au moment de la diffusion du film 4 .

Les marqueurs du style du reportage filmique sont ici mobilisés en vue de captiver l'attention du public et de démystifier la représentation héroïque de la guerre. Un bref détour par les prises de position théoriques de Watkins sur les médias permet de déterminer plus généralement ses intentions ou son mythe expressif, auxquels ses films ne se laissent pas pour autant réduire.

\section{La Monoforme, ou la "théorie critique " à l'époque du spectaculaire intégré}

Watkins dénonce inlassablement la logique, l'économie et les méthodes qui prévalent dans la construction de l'information télévisée et de l'industrie du cinéma dominant, stigmatisant les "mass media audiovisuels» comme soumis à une "Monoforme» $\mathbf{5}$. Cette critique radicale, il l'a d'abord formulée à l'occasion de conférences prononcées dans des lycées et des universités, entre 1968 et 1977, vis-à-vis de sa propre
4 Lors de la première diffusion de Culloden sur la BBC, le caractère abrupt de la représentation de la bataille est souligné tant dans les critiques positives ("Bitter, Brutal, Brillant", Daily Record and Mail, 16 décembre 1964; "The Horrible Reality of the Last Battle in Britain ", Daily Mail, 16 décembre 1964; Mary Crozier, "Culloden on BBC-1", Guardian, 16 décembre 1964; Stewart Lane, "What a Savage Indictment!", Daily Worker, 19 décembre 1964; Roy Wilson, "Grim, Gruesome and Impressive", Eastern Evening News, 16 décembre 1964) que dans les attaques portées contre le film ("Protest at "Sadistic, Culloden TV Show", Birmingham Evening Mail, 17 décembre 1964). De la crudité dans la représentation à la cruauté dans la direction des acteurs, il n'y a qu'un pas allégrement franchi par le syndicat des acteurs britanniques Equity, qui dénonce la maltraitance des interprètes amateurs, manipulés "pour les besoins du réalisme" du film ("Producer Denies Extras Were Exhausted", Times, 21 décembre 1964). Voir James Michael Welsh, Peter Watkins. A Guide to References and Resources, Boston, G.K. Hall, 1986, pp. 66-69.

5 Voir notamment Peter Watkins, "The MAVM and Globalization", http://pwatkins.mnsi.net/ appendix_2.htm. 
6 En 2003, Watkins met en ligne sur son site internet ses réflexions sur les mass media, à travers un texte pamphlétaire qu'il intitule Media Crisis [voir http://pwatkins.mnsi.net/ part1_home.htm]. La traduction française de cet essai est publiée en 2004 (Peter Watkins, Media crisis, Paris, Editions Homnisphères, 2004); nous citons la seconde édition française augmentée, établie en 2007.

7 Id., p. 36. Et Watkins de préciser: "Il existe plusieurs variantes de la Monoforme: la structure narrative mono-linéaire classique, utilisée dans les films de cinéma, les sitcoms et les feuilletons policiers; le mélange fluide de thèmes et d'images apparemment décousues, propre aux chaînes de télévision musicales telles que MTV; la structure saccadée et fragmentaire des informations télévisées du monde entier ainsi que de nombreux documentaires [...]. Ces variantes de la Monoforme ont des caractéristiques communes: elles sont répétitives, prévisibles, et fermées à toute participation des spectateurs." (id., p. 37).

8 Voir Theodor W. Adorno, Max Horkheimer, "La production industrielle des biens culturels ", La dialectique de la raison, Paris, Gallimard, 1974 [Philosophische Fragmente, New York, Social Studies Association, 1944; Dialektik der Aufklärung: philosophische Fragmente, Amsterdam, Querido, 1947].

9 /d., p. 163.

10 "L'emploi permanent de la Monoforme avec son absence d'espace de réflexion, son entrain narratif (difficilement détectable tant elle paraît fluide), sa progression monolinéaire et ininterrompue (qui fige ainsi la mémoire en déniant toute complexité à l'expérience humaine) - a eu d'évidentes et d'incalculables conséquences à long terme sur nos émotions. [...] Par sa capacité de fragmentation et de division, cette forme narrative génère de fortes impulsions anti-démocratiques au sein du processus social. Le manque d'inclination, si répandu dans nos sociétés occidentales, pour toute forme d'engagement collectif, au profit de son contraire (des comportements de plus en plus égoïstes, auto-centrés et la privatisation de la sphère publique), n'est que l'une des manifestations des effets sous-jacents à long terme de la Monoforme." (Peter Watkins, Media crisis, op. cit., pp. 40-41).
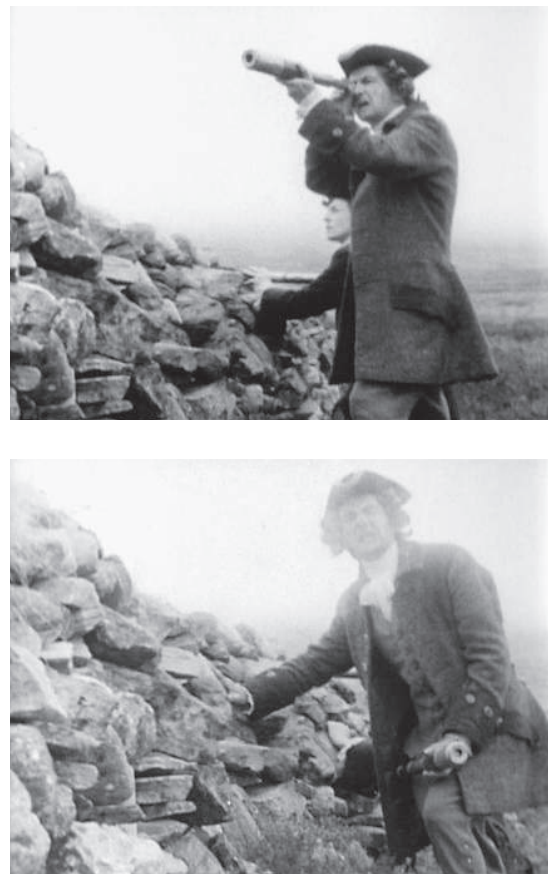

pratique filmique, en premier lieu The War Game (La bombe, 1965). Il l'a systématisée, à l'occasion du séminaire qu'il a animé à Columbia University en 1979, en analysant avec les étudiants la structure des journaux télévisés. En 2003, il recueille ses réflexions dans un essai incisif destiné à un large public 6 . Condamnant le «dispositif narratif interne» de la "Monoforme» en tant que «mitraillage dense et rapide de sons et d'images» à travers une «structure, apparemment fluide mais structurellement fragmentée 》 7 , il réactualise par rapport à la sphère télévisuelle la radicalité de la «théorie critique» telle qu'elle avait été formulée par Horkheimer et Adorno à l'encontre de l'industrie des loisirs audio-visuels, en 19448. En effet, ceux-ci comparent les films des grands studios américains ou encore les tubes en vogue à la production en série de clefs Yale ou au montage en ligne d'automobiles 9 , tandis que celui-là rapporte le formatage des durées à la télévision au mécanisme réifiant de l'«Horloge universelle» et la mise en forme des sujets à une décontextualisation des propos suivant un rythme saccadé10. La charge est polémique chez Watkins, mais l'argumentaire peut paraître peu dialectisé, absolutisant le schème de l'aliénation sans possibilité de dépassement ou de renversement. Watkins, il est vrai, a vécu concrète- 

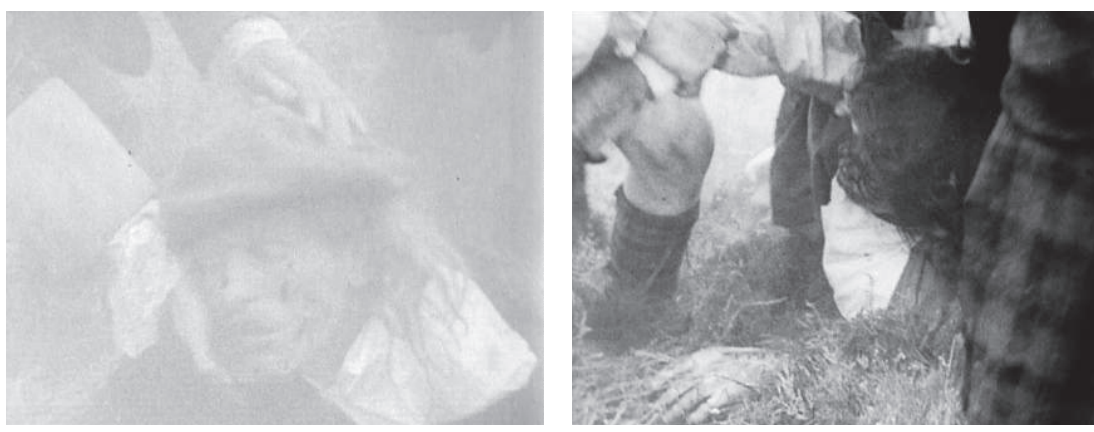

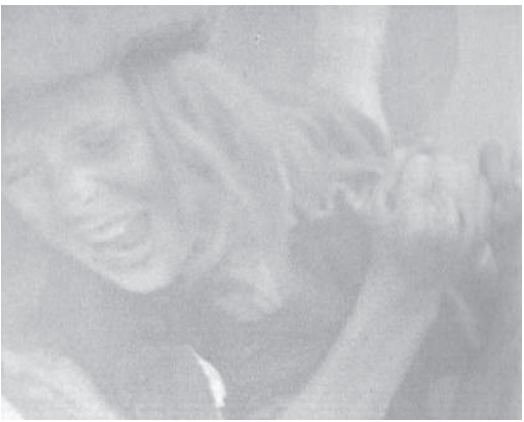

ment l'expérience de la censure (directe, ou plus médiate) exercée par la télévision et plus généralement par les réseaux de production et de diffusion cinématographiques. Mais ce qu'il vise, dans une perspective plus "dialectique» que la dénonciation unilatérale de la "Monoforme", avec The War Game (une production de la BBC de 1965, simulant une attaque nucléaire contre l'Angleterre), puis avec Punishment Park (une autoproduction de 1970, mettant en scène la répression des opposants américains à la guerre du Vietnam) ou encore avec La Commune (une coproduction d'Arte et 13-Production de 1999, représentant l'insurrection révolutionnaire de 1871 à Paris), revient précisément à constituer un «espace public oppositionnel» $\mathbf{1 1}$ au sein même des mass media ou de la branche cinématographique. Que ce geste d'insurrection se solde par un échec (une interdiction de diffusion télévisuelle pendant vingt ans, une sortie marginale en salles $\mathbf{1 2}$ et une diffusion unique en fin de soirée sur Arte) constitue un fait qui n'invalide pas pour autant la logique présidant à cette tentative de subversion de l'institution depuis ses propres canaux de communication. Si le dispositif de monstration imaginé par Peter Watkins pour The War Game n'a pas pu être actualisé, son efficacité peut néanmoins être évaluée sur le plan théorique: le téléspectateur,
11 Oskar Negt et Alexander Kluge critiquent la notion d'"espace public" théorisée par Habermas (Jürgen Habermas, L'espace public. Archéologie de la publicité comme dimension constitutive de la société bourgeoise, Paris, Payot, 1978 [Strukturwandel der Öffentlichkeit. Untersuchungen zu einer Kategorie der bürgerlichen Gesellschaft, Berlin, Luchterhand, 1962]) comme une plate-forme d'expression bourgeoise qui se fonde sur l'exclusion du prolétariat et des femmes. A l'opposé, ils conceptualisent un "espace public oppositionnel " (Oskar Negt, Alexander Kluge, Öffentlichkeit und Erfahrung. Zur Organisationsanalyse von bürgerlicher und proletarischer Öffentlichkeit, Francfort, Suhrkamp, 1972 [Public Sphere and Experience. Toward an Analysis of the Bourgeois and Proletarian Public Sphere, Minneapolis/Londres, University of Minnesota Press, 1993]). A notre connaissance, Peter Watkins ne mobilise pas ce modèle théorique qui permet pourtant de décrire précisément sa stratégie médiatique et qui imprègne les études littéraires et culturelles anglo-saxonnes depuis les années 1990, les actions des avant-gardes étant réévaluées dans une perspective féministe à partir de cet espace de parole ouvert, multiple et non hiérarchisé.

12 Punishment Park, présenté au New York Film Festival en octobre 1971, est retiré de l'affiche après quatre jours d'exploitation dans une salle excentrée à New York; il sera encore diffusé dix jours dans un cinéma de San Francisco (voir Peter Watkins, "Punishment Park and Dissent in the West" [octobre 1973], Literature/Film Quarterly, vol. 4, n 4, automne 1976, pp. 293302). En France, le film sort en salles, mais est interdit aux moins de 18 ans. Le film est réalisé avec l'appui de l'organisation des Etudiants pour une société démocratique (SDS), et se heurte à sa sortie à l'opposition de la fédération des Jeunes républicains (YR). 
13 Le récit est peut-être apocryphe, mais les responsables de la chaîne Granada TV auraient refusé de diffuser Forgotten Faces (1960), à cause du "réalisme exacerbé " de cette reconstruction de l'insurrection de Budapest de 1956; suite à sa vision, l'un des directeurs de la chaîne aurait déclaré: "plus personne ne croira en nos journaux télévisés "... (Milton Shulman, "Inside TV: Why Did They Play The War Game Without Me?", Evening Standard, 16 février 1966, cité par James M. Welsh, Peter Watkins, op. cit., pp. 99-100).

14 Depuis les années 1970, le cinéma de Peter Watkins est systématiquement associé au genre de la "politique-fiction" (variation ou plutôt dérivation à partir du mot composé "science-fiction") dans la littérature secondaire. Ainsi, dans Trente ans de cinéma britannique, Watkins est-il présenté comme un expert en la matière: "En quatre films, Peter Watkins est devenu un spécialiste du film de politiquefiction. Chaque fois, Peter Watkins pose un postulat de départ et répond à la question: 'Qu'arriverait-il si...). Systématiquement, il pousse l'argumentation dans ses derniers prolongements et, le plus souvent, donne à son propos la forme d'une émission tournée en direct." (Raymond Lefèvre, Roland Lacourbe, Trente ans de cinéma britannique, Paris, Editions Cinéma 76, 1976, p. 288).

15 Luc Boltanski, Eve Chiapello, Le nouvel esprit du capitalisme, Paris, Gallimard, 1999.

$16 / d .$, p. 82. en découvrant l'émission de Watkins, s'interroge non seulement sur les conséquences entraînées par un conflit nucléaire, mais encore sur la codification de la représentation dans les reportages et les actualités $\mathbf{1 3}$. La critique des mass media s'exerce en ce cas à travers le recours même aux médias, la diffusion du "reportage de politique-fiction"sur les chaînes publiques amplifiant l'ambivalence de son énonciation, le téléspectateur oscillant entre un mode de lecture documentarisant et fictionalisant, entre un contrat de lecture réaliste (prendre l'information à la lettre) ou distancié (considérer le reportage comme une parabole). Nous reviendrons sur les caractéristiques de la "politique-fiction", clef d'entrée régulièrement convoquée pour restituer la logique des films de Watkins ${ }^{\mathbf{1 4}}$. Mais il convient d'emblée de souligner l'usage authentiquement politique qu'il en fait: sa démarche filmique repose sur un geste d'appropriation de marqueurs de genres audio-visuels (le reportage télévisuel pour The War Game et Punishment Park), qui sont mis en jeu dans un univers «étrangéifié », les codes cités étant dès lors défamiliarisés, mis en scène dans leur artificialité même.

\section{Critique artiste, critique sociale, et la New Left américaine}

Indéniablement, un souci politique anime Peter Watkins : tant son travail cinématographique que ses interventions écrites peuvent être qualifiés de "critiques». Nous souhaiterions ici tenter d'identifier plus précisément le type de "critique» exercé par Watkins. Dans leur ouvrage Le nouvel esprit du capitalisme ${ }^{15}$, Luc Boltanski et Eve Chiapello, en analysant les événements contestataires survenus en France dans les années 1960, identifient deux modalités principales de critiques, soit la "critique artiste» et la "critique sociale». Ces "critiques» s'opposent toutes deux au capitalisme. Toutefois, elles ne s'en prennent pas aux mêmes aspects. La critique artiste est une dénonciation du capitalisme comme "source de désenchantement et d'inhautenticité des objets, des personnes, des sentiments et, plus généralement, du genre de vie qui lui est associé", ainsi que du capitalisme comme "source d'oppression, en tant qu'il s'oppose à la liberté, à l'autonomie et à la créativité des êtres humains [...]». La critique sociale, de son côté, s'attaque au capitalisme comme "source de misère chez les travailleurs et d'inégalités [...]», ainsi qu'au capitalisme comme "source d'opportunisme et d'égoïsme»16. La première "critique" est d'ordre éthico-esthétique. Elle se focalise sur des enjeux de modes de vie, dénonce l'aliénation capitaliste qui empêcherait les individus d'exprimer leur nature créative, ou combat la répression de toute organisation alternative de la vie personnelle ou communautaire. Le consumérisme et la course au profit sont stigmatisés davantage 
pour leur aspect aliénant et normalisateur que pour leurs conséquences économiques. Cette "critique" appelle à une organisation autonome des individus, à une fondation horizontale des interactions humaines, à l'autogestion. La seconde est de type économique, s'attachant à la question de la répartition des richesses et à celle de la pauvreté, rejetant les aspects inégalitaires du capitalisme et critiquant les conséquences néfastes, sur la population ouvrière et artisane, de l'enrichissement d'un nombre restreint de personnes. Elle appelle à une répartition équitable des richesses, à une mise en commun des ressources.

Différentes quant à leur objet, ces deux "critiques» ont été défendues par des mouvements et des individus distincts. La critique artiste se retrouve notamment dans le surréalisme ${ }^{\mathbf{1 7}}$, le freudo-marxisme et l'Ecole de Francfort ${ }^{\mathbf{1 8}}$, le situationnisme ${ }^{\mathbf{1 9}}$ ou la philosophie de Deleuze et Guattari 20 - elle est principalement fondée dans les philosophies allemande et française et dans les mouvements d'avant-garde ou estudiantins. La critique sociale est mobilisée par le marxisme traditionnel, mais aussi par les partis politiques de gauche et les syndicats - son ancrage est moins déterminable géographiquement, puisqu'elle est ipso facto assumée par toute organisation politique anti-capitaliste institutionnelle. Dans quelle tradition s'inscrit donc Peter Watkins?

Dans Edvard Munch (1973), la vie de bohème est fortement présente. Le film retrace la vie et les activités artistiques du jeune Munch à Kristiania et à Berlin, pendant les années 1882-1895, soit avant sa reconnaissance publique ${ }^{21}$; le monde prolétaire (les ouvriers, mais aussi les prostituées) est systématiquement opposé au monde bourgeois (les couples déambulant sur l'avenue Karl Johan, la figure autoritaire du père), Munch adhérant aux prises de position de l'anarchiste Hans Jaeger. Cette Bohème historique, qui appelle à "vivre autrement», s'inscrit dans la critique "artiste». En outre, dans les textes théoriques de Watkins, certains passages font référence à des thématiques associées à ce type de «critique». Le rejet par Watkins de la Monoforme est notamment motivé par le fait qu'elle atomise les rapports sociaux, séparant les individus les uns des autres et les empêchant de se réunir en collectivité, alors qu'ils tendent "authentiquement» à cela. C'est en ce sens aussi qu'il peut dénoncer l'action néfaste des médias:

"Ce climat [audiovisuel] [...] a entraîné chez nous une sérieuse diminution de notre capacité de concentration, un manque de tolérance pour des processus soutenus ou pour n'importe quelle forme de communication qui exigerait d'y consacrer plus de dix secondes, une amnésie de plus en plus généralisée face à notre histoire $[. .$.$] , un besoin perpétuel et accru de changements. Tout cela$
17 La volonté surréaliste de faire éclater la conscience pour laisser émerger la spontanéité poétique a toujours été accompagnée d'un appel à vivre autrement, la forme de vie bourgeoise par trop "rationnelle" ne pouvant faire place à cet inconscient poétique. Le personnage de Nadja d'André Breton (Paris, Gallimard, 1963 [première publication: Paris, NRF, 1928]) ne fait que radicaliser la vie de rupture que le surréalisme revendique. L'engagement communiste de ses membres n'avait pas d'autre sens (du moins dans les années 1920).

18 Le freudo-marxisme prend sa source dans l'essai de Wilhelm Reich, "Matérialisme dialectique et psychanalyse" (repris dans La crise sexuelle, Paris, Editions sociales internationales, 1934 ["Dialektischer Materialismus und Psychoanalyse", Unter dem Banner des Marxismus, vol. 3, 1929, pp. 736-771]). Cette pensée, qui ajoute à la critique économique du capitalisme une critique de la répression bourgeoise de la libido, a fortement influencé les penseurs de l'Ecole de Francfort, notamment Marcuse (Herbert Marcuse, L'homme unidimensionnel, Paris, Editions de Minuit, 1968 [Onedimensional Man, Boston, Beacon, 1964]). Le texte inaugural de l'Ecole de Francfort est $L a$ dialectique de la raison de Max Horkeimer et Theodor W. Adorno (op. cit.).

19 Si La société du spectacle de Guy Debord (Paris, Buchet-Chastel, 1967) constitue le versant critique du situationnisme, on trouve son versant positif dans le fameux Manuel de savoir-vivre à l'usage des jeunes générations de Raoul Vaneigem (Paris, Gallimard, 1967).

20 C'est principalement dans L'Anti-CEdipe (Paris, Editions de Minuit, 1972) et Mille Plateaux (Paris, Editions de Minuit, 1980), les deux volumes de Capitalisme et schizophrénie, que Deleuze et Guattari élaborent leur théorie d'opposition au capitalisme comme système de répression du désir.

21 Voir Michèle Lagny, Edvard Munch (Peter Watkins), Lyon, Aléas, 2011, p. 44. 
a permis de façonner une société manifestement plus privatisée, où règnent l'insécurité et une agitation constante. Une société où la pensée compétitive, l'égotisme, le gain personnel, et l'indifférence envers la violence et la souffrance deviennent de plus en plus <la norme > et où disparaissent la pluralité authentique et l'interaction communautaire.»22

L'usage d'une notion telle que celle de "pluralité authentique» (genuine plurality), la revendication d'une organisation par le bas des relations sociales et la critique des médias comme facteur de division des individus, rappellent indéniablement les thématiques phare de la «critique artiste».

Mais la présence de cet esprit libertaire chez Watkins ne semble pas exclure la "critique sociale", ses films mettant également en scène les luttes syndicales et ouvrières anti-capitalistes. La Commune (Paris, 1871) constitue ainsi un véritable plaidoyer en faveur de l'autogestion et de l'organisation en conseils ouvriers, l'épisode insurrectionnel de 1871 étant relu à travers les événements de Mai 68. De façon moins explicite, les luttes ouvrières sont également au centre d'Evening Land (Force de frappe, 1976). Watkins relate dans ce film de "politique-fiction" la situation d'une grève générale au Danemark, due au refus des ouvriers de collaborer à la construction d'armes nucléaires, ainsi que l'enlèvement d'un ministre par un groupuscule terroriste de gauche qui soutient la grève. Ce film dénonce le marché de l'armement, sans que son propos ne se limite pour autant à cette accusation. Plus que le contenu thématique d'Evening Land, ce qui prime, c'est son dispositif filmique, en ceci que le cinéaste modifie son centre de focalisation. Durant la majeure partie du film, Watkins opte pour la forme journalistique-télévisuelle: il montre des événements dans leur succession causale et décrit de façon cursive une crise socio-politique allant crescendo. Certaines scènes, par contraste, rompent avec le caractère documentaire du film, telles celles où les terroristes sont filmés durant leurs délibérations - un journaliste n’y aurait pas accès. Toutefois, nous pensons que le filmage depuis le groupuscule terroriste ne remplit pas uniquement cette fonction d'«impression de fictionalité». Le but est plutôt l'assimilation du cinéaste aux militants d'ultra-gauche. Autrement dit, l'énonciateur du film s'identifie aux activistes, en reprenant à son compte le discours contestataire des terroristes de gauche. Cette superposition entre énonciation filmique et énonciation militante nous semble explicitée dans une scène centrale du film, qui se déroule dans le quartier résidentiel bourgeois de Svanemöllevej, à Copenhague. Depuis l'intérieur d'une voiture, contexte intimiste, la caméra filme une petite rue comprenant divers carrefours. En voix 
over, une membre du groupuscule lit un message politique destiné à être rendu public:

«Nous avons donné au gouvernement une liste des endroits où nous voulons agir d'une façon ou d'une autre. De plus, nous avons vingttrois objectifs dont nous n'avons rien dit. Nous espérons disperser la police le plus possible pour qu'il soit impossible aux dirigeants d'intervenir pour empêcher la grève illégale.»

La voiture roule lentement dans la rue et la caméra, portée à l'épaule, filme depuis le siège arrière du véhicule une rue sur la droite, zoomant en direction de policiers postés plus loin. Cette scène est entrecoupée par une harangue à l'usine, un syndicaliste s'adressant à la foule :

"Il y a de quoi s'inquiéter quand on nous montre à la télé comment la police danoise se rue sur les groupes soi-disant de gauche... [la foule: "A bas les fascistes!»] ... sous prétexte qu'ils espèrent trouver des complices ou des sympathisants du groupe qui a enlevé le ministre.»

Le film revient à la scène de la voiture, dont le silence, uniquement rompu par la voix over, contraste fortement avec la scène de harangue. L'opérateur filme à nouveau une rue sur la droite et zoome en direction des policiers. La caméra panote ensuite sur la gauche, cadrant le conducteur, puis continue à balayer le champ, avant de zoomer à nouveau en direction des policiers. Or, dans le champ, au premier plan, apparaît cette fois le visage d'une femme, assise dans la voiture à côté de l'opérateur, tenant un micro à la main et lisant le texte qui semblait émaner du hors-champ. La voix over s'ancre rétroactivement dans le champ, le texte étant visiblement lu par la passagère du véhicule:

"Nous sommes dans le quartier de Svanemöllevej où habitent quatre hommes avec leur famille. C'est le directeur de la Banque privée,

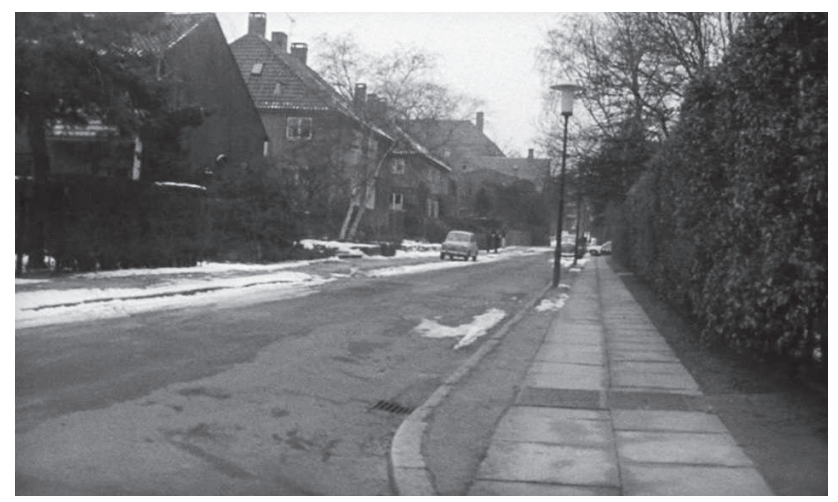


l'ambassadeur d'Allemagne, l'attaché militaire belge, et le P.-D.G. d'IBM. Nous avons prévenu le gouvernement que nous voulions agir d'une façon ou d'une autre contre ces hommes. Nos menaces ont mis la répression au grand jour et tout le monde peut la voir. La police a pris position partout où le pouvoir est concentré. Sortez tous et regardez-les bien.»

Entre-temps, la caméra, à l'épaule toujours, balaye le champ depuis la gauche et filme l'extrémité de la rue dans laquelle la voiture s'avance, où sont également postés des policiers vers lesquels l'opérateur zoome à nouveau. Le plan suivant montre un représentant du gouvernement qui s'adresse aux journalistes, sur fond d'une musique soulignée.

Ainsi, frayant à droite, à gauche, puis s'avançant frontalement, la caméra, tout comme les militants - encerclés par les policiers, par «l'Etat répressif»-, constituent une communauté à part, radicale, marginale, qui balaye le champ du pouvoir des yeux, contrôlant la situation. La voiture et ses occupants, dont les visages sont difficiles à discerner, constituent «l'espace public oppositionnel» de Watkins, le film s'apparentant à un acte politique. Acteurs et opérateur sont placés au même niveau, tous assis dans la voiture, et la voix over est destituée de son rôle métanarratif pour redescendre au niveau de l'action même. Il y a écrasement ou superposition entre le point de vue de l'équipe de tournage et celui de l'action. Les comédiens nous montrent ainsi qu'ils ont feint de jouer la comédie, tandis que Watkins a simulé la position "dominante» du cinéaste de fiction. Ce qui motive son geste, c'est de prendre position au sein même du discours militant, d'en assumer le contenu - mais pas nécessairement la violence -, bref, de s'identifier aux activistes. Ainsi, acteurs et cinéastes sont destitués de leur identité et de leurs fonctions cinématographiques pour fonder une communauté dans laquelle filmer
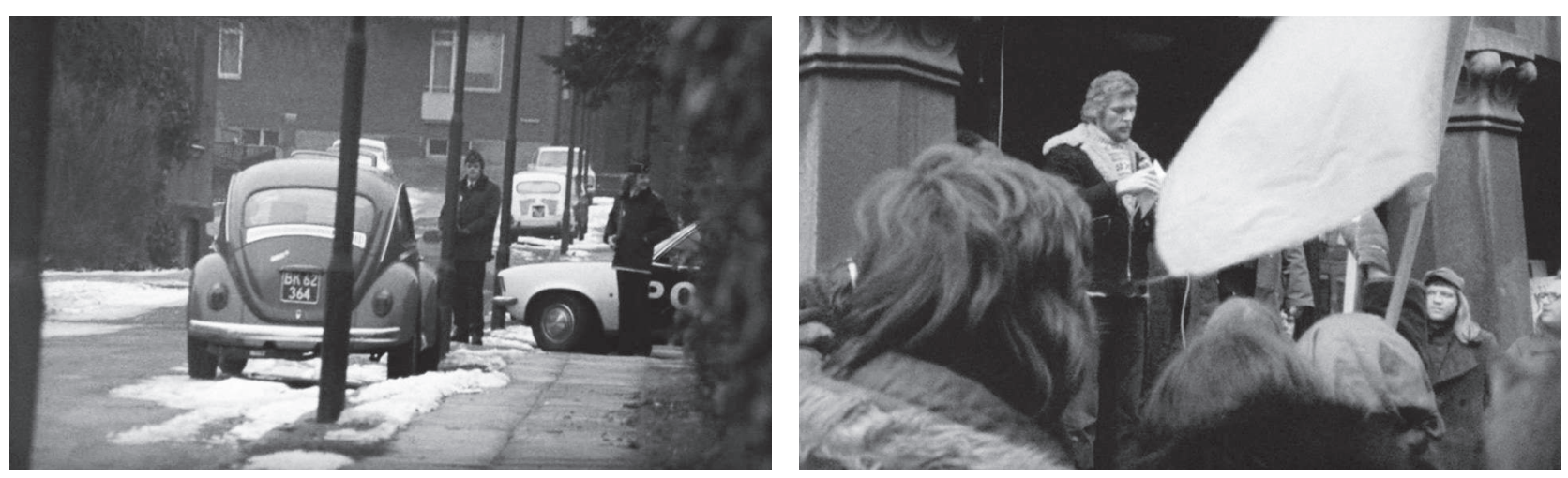
et assumer un discours ne se distinguent plus, dans laquelle tenir le rôle du réalisateur ou de l'acteur s'indifférencient, tout étant subsumé sous l'activité politique. Cette constitution d'un espace communautaire alternatif repose sur une structure organisée horizontalement et qui n'est pas sans évoquer les principes de l'autogestion. La "critique» chez Watkins est encore performative: filmer les militants, c'est militer. Nous développerons plus longuement les liens de Watkins à la performance et à la performativité, qui nous semblent constituer tout particulièrement l'intérêt et l'ambiguïté de ses films.

Mais revenons au propos militant de Watkins, et tentons d'identifier historiquement son discours. Malgré la grande attention portée à l'organisation ouvrière et à l'activisme de gauche dans Evening Land, l'anti-militarisme est, comme souvent chez Watkins, le motif principal de la contestation. Les défilés de dignitaires militaires européens sur tapis rouge rappellent ceux de The Gladiators (1968), la menace militaire et nucléaire les horreurs de Culloden et de The War Game. L'objet de la critique est finalement moins le capitalisme que la guerre et la course à l'armement. Cette focalisation sur la question de l'armement nucléaire éloigne Watkins du cinéma militant ouvrier, par exemple de celui des Groupes Medvedkine 23. Ainsi, s'il fallait identifier historiquement la critique présente dans les films de Watkins, le point d'ancrage serait très vraisemblablement la New Left américaine $\mathbf{2 4}$ : critique du militarisme et de la course à l'armement, critique de l'autoritarisme d'Etat, critique de la répression contre les pacifistes. Bien que les valeurs de la New Left ne soient pas étrangères aux mouvements d'avant-garde d'Europe continentale (autogestion et libertarisme), ni aux luttes ouvrières, la focalisation sur le militarisme constitue néanmoins un signe distinctif de la contestation américaine. Ceci s'explique par l'importance de
23 Voir notamment Emmanuel Barot, Camera Politica: dialectique du réalisme dans le cinéma politique et militant (Groupes Medvedkine, Francesco Rosi, Peter Watkins), Paris, Librairie philosophique J. Vrin, 2009; Sébastien Layerle, Caméras en lutte en Mai 68. "Par ailleurs le cinéma est une arme", Paris, Nouveau Monde, 2008.

24 Voir notamment Van Gosse (éd.), The Movements of the New Left, 1950-1975: a Brief History with Documents, Boston, Bedford/ St. Martin's, 2004; Maurice Isserman, Michael Kazin, America Divided: the Civil War of the 1960s, Oxford, Oxford University Press, 2000; Priscilla Long (éd.), The New Left: a Collection of Essays, Boston, P. Sargent, 1970.
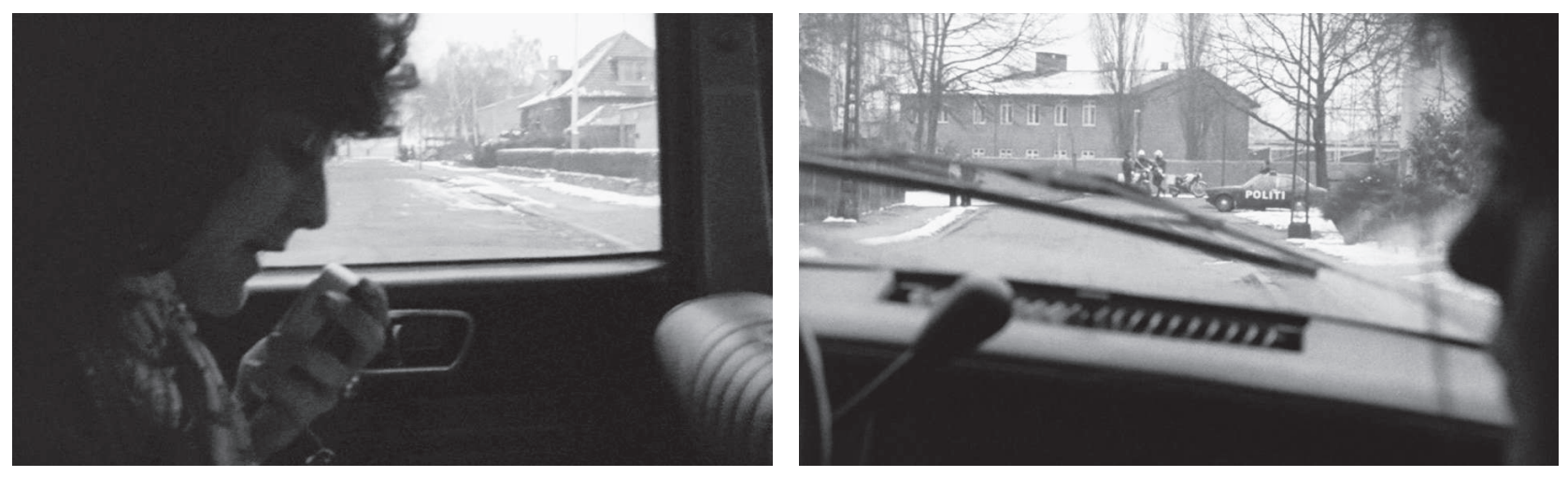

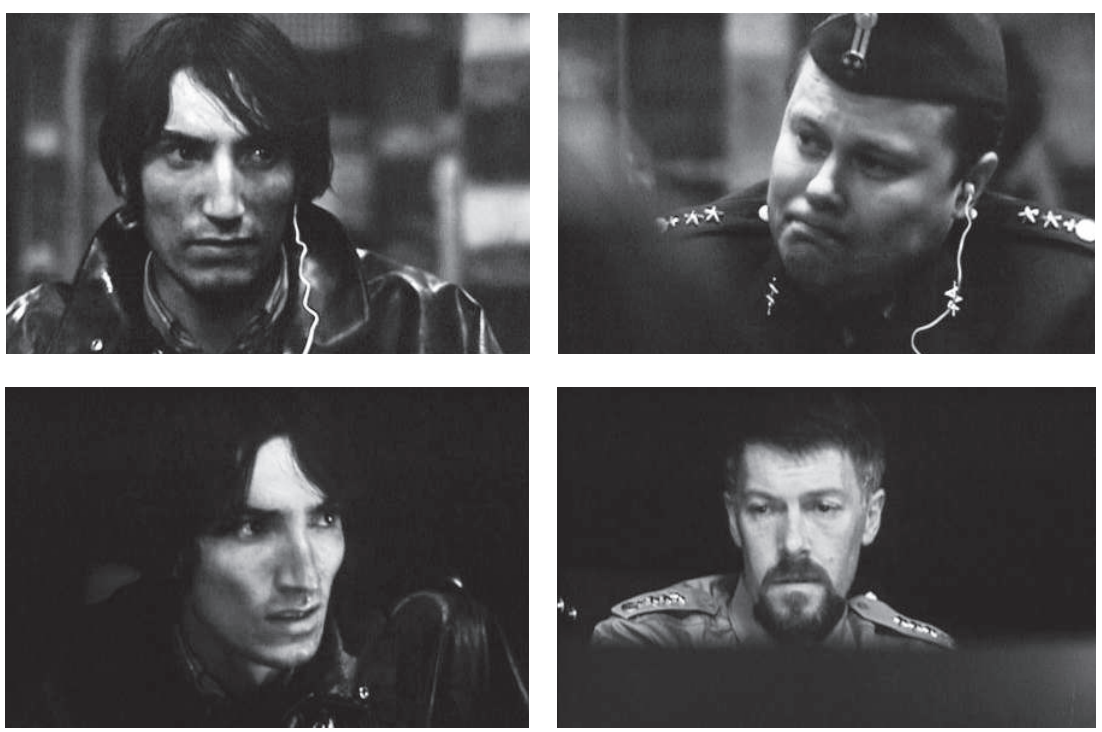

l'opposition à la guerre du Vietnam, qui n'a pas tant modelé les luttes sur le continent qu'elle ne l'a fait aux Etats-Unis, parmi les étudiants, les intellectuels et les militants. C'est indéniablement dans cette tradition que s'inscrit Watkins. La «critique artiste» et la "critique sociale» chez Watkins sont, le plus souvent, au service d'une critique de l'armement et de la violence militaire d'Etat. En cela, Watkins propose au spectateur une monomanie parfois dérangeante. Pour qui souhaite qualifier l'impérialisme militaire d'épiphénomène du capitalisme, de son extension "par d'autres moyens", Evening Land ne peut que sembler manquer sa cible, la peur de la catastrophe nucléaire prenant le pas sur l'analyse des rapports de classe.

Cette focalisation sur la guerre devient délirante dans The Gladiators, film tourné en $35 \mathrm{~mm}$ qui se situe à l'orée de la science-fiction. Rappelons son scénario. Les grandes puissances militaires mondiales ont décidé de s'opposer, à armes réelles mais via des équipes de mercenaires, dans un jeu télévisé, cette médiation «ludique» de la guerre étant destinée à sublimer les penchants destructeurs de l'humanité. L'anti-militarisme de Watkins prend ici fortement le dessus. Toutefois, la critique du capitalisme est également présente dans The Gladiators, bien qu'elle y occupe une place plus faible que dans ses écrits théoriques. En effet, parallèlement au combat mené par les mercenaires des différentes armées, un intrus parvient à entrer dans l'enceinte de combat - une propriété en rase campagne -, dans le but de prendre possession de la machine Icarus, qui gère le jeu 
et plus généralement le «système». Cet intrus, qui s'exprime en français, tient un discours d'étudiant-militant de Mai 68 : mise à niveau du travail manuel et intellectuel, partage des richesses, etc. - le film datant de 1969, l'allusion historique est évidente. La scène la plus explicite à cet égard est un champ/contrechamp conventionnel entre l'étudiant français et l'adjoint du contrôleur d'Icarus, rencontré à la fin du périple du jeu de la guerre. Les protagonistes, assis face à face, se parlent par l'intermédiaire d'Icarus qui traduit du français et du suédois. L'adjoint sourit d'un air narquois pendant le discours politique de l'étudiant, et regarde parfois la caméra comme pour décrédibiliser davantage la vraisemblance de la scène, sur fond d'une musique indienne décalée. Ce face à face quelque peu dérisoire entre Mai 68 et le pouvoir répressif est reconduit dans la scène finale du film. L'étudiant, parvenu à la salle de contrôle, est cadré de face, assis à côté de l'administrateur d'Icarus, tous deux se tenant devant le tableau de commande de la machine. L'administrateur lui explique que lui aussi, autrefois, avait des idéaux politiques qui l'avaient mené jusqu'à la salle de contrôle, mais qu'une fois en possession de la machine, celle-ci avait pris le dessus. Dans un discours d'un pessimisme burlesque, il prédit un dénouement identique en ce qui concerne l'action de l'étudiant. On peut se demander, ici, si le discours contestataire est pris au sérieux, car il s'apparente plutôt à une sorte de farce cryptofreudienne sur les penchants guerriers de l'humanité. Mais ce serait là se cantonner à une analyse des thèmes et des situations mis en scène dans les films de Watkins, tandis que leur propos critique se déploie à travers l'élaboration collective d'un dispositif performatif qui emprunte le plus souvent un mode d'énonciation conditionnel, hypothétique.

\section{L'impulsion allégorique de la "politique-fiction"}

Le caractère traumatique de l'œuvre, activement recherché par Watkins, ne se rapporte pas tant, selon nous, à une entreprise de déconstruction et de critique des médias de masse, qu'à une "impulsion allégorique $\mathbf{2 5}$ qui sous-tend l'univers de "politique-fiction" mis en scène. Le cinéma de Watkins met en jeu un mode d'énonciation contradictoire: le geste d'appropriation de conventions télévisuelles qui concourent à un «effet de réel " entre en tension avec la logique de dramatisation et de généralisation qui est associée à l'hypothèse de "politique-fiction" (se traduisant paradoxalement par la symbolicité indéterminée de l'univers filmique lorsqu'il est projeté dans un futur proche et par l'authentification des faits lorsqu'il s'agit de reconstituer un événement historique). Le genre de la "politique-fiction", tel qu'il s'actualise dans les films de Watkins, peut être décrit comme un geste de "feintise» $\mathbf{2 6}$ (ou de trompe-l'œil,
25 Benjamin, dans son analyse du drame baroque allemand, met en évidence une logique allégorique qui procède par fragments (voir Walter Benjamin, Origine du drame baroque allemand, Paris, Flammarion, 1974 [Ursprung des Deutschen Trauerspiel, Berlin, Ernst Rowohlt Verlag, 1928]). Owens réactualise ce modèle dans le but de rendre compte de la logique de l'Appropriation Art qui émerge à la fin des années 1970: "Dans la structure allégorique, donc, un texte est lu à travers un autre, aussi fragmentaire, intermittente ou chaotique leur relation puisse-t-elle être; le paradigme de l'œuvre allégorique est, par conséquent, le palimpseste. [...] L'imagerie allégorique est une imagerie que l'on s'approprie; l'allégoriste n'invente pas les images, il les confisque. II s'attribue le signifiant culturel, se pose comme son interprète." (Craig Owens, "L'impulsion allégorique: vers une théorie du postmodernisme", dans Charles Harrison et Paul Wood, éd., Art en théorie, 1900-1990, Paris, Hazan, 1997, p. 1147 ["The Allegorical Impulse: Toward a Theory of Postmodernism ", October, $\mathrm{n}^{\circ} 12$, printemps 1980 , pp. 67-86; October, n० 13 , été 1980, pp. 58-80]).

Ce mouvement d'appropriation des représentations pourrait être transposé par rapport à l'univers des reportages de "politique-fiction" de Watkins, pour autant que I'on introduise comme troisième terme la théorie du complot développée par Fredric Jameson (La totalité comme complot: conspiration et paranoïa dans l'imaginaire contemporain, Paris, Les Prairies ordinaires, 2007 [The Geopolitical Aesthetic: Cinema and Space in the World System, Bloomington, Indiana University Press, 1992]).

26 Sur la feintise en tant que genre littéraire, voir John R. Searle, "Le statut logique du discours de la fiction ", Sens et expression: études de théorie des actes du langage, Paris, Minuit, 1982 ["The Logical Status of Fictional Discourse", Expression and Meaning: Studies in the Theory of Speech Acts, Cambridge, Cambridge University Press, 1979]; Käte Hamburger, Logique des genres littéraires, Paris, Seuil, 1986 [Die Logik der Dichtung, Stuttgart, Klett, 1957]. Jean-Marie Schaeffer décrit en ces termes le pacte de lecture qui caractérise le mode de la "feintise partagée": "Nous ne savons pas si une chose est vraie ou réelle, mais nous la croyons comme telle." (JeanMarie Schaeffer, Pourquoi la fiction?, Paris, Seuil, 1999, p. 199). 
27 Propos de Watkins rapportés par James Green, "It Costs $£ 10$ '000 - But You'll Never See It!", Evening News, 26 novembre 1965 (cité par James M. Welsh, Peter Watkins, op. cit., pp. 79-80). Dans le même article, Huw Weldon précise: "Nous savions qu'il était risqué de vouloir réaliser un documentaire réaliste sur les effets possibles d'une guerre nucléaire. Mais à cause de l'extrême importance du sujet, nous avions jugé qu'il valait la peine de tenter une telle entreprise. La décision de diffuser ou non le résultat a été extrêmement difficile à prendre.".

28 Peter Watkins, "Un Anglais en colère", Cinéma 67 - Image et son, n 116 , mai 1967 , p. 11.

29 Notre analyse de la réception critique francophone des films de Watkins repose principalement sur les coupures de presse réunies à la Cinémathèque suisse. Pour la réception critique anglo-saxonne, nous nous en remettons à la bibliographie raisonnée établie par James M. Welsh (Peter Watkins, op. cit.). Dans la littérature secondaire sur Watkins, la réception critique n'est que rarement analysée. Nous le faisons ici pour la Suisse romande et dans une moindre mesure pour la France, contribuant modestement à pallier l'absence de ce type d'analyses, qui permet pourtant de contextualiser les œuvres et de révéler les réactions qu'elles ont suscitées.

30 Voir Jacques Isnard, "La bombe, un film de réalité-fiction ", Le Monde, 7 avril 1967; Charlotte Hug, "La bombe. Une terrible illustration de réalité-fiction " ", Construire, 4 octobre 1967.

31 Voir Michel Buenzod, "Machinalement, la guerre thermonucleaire", Bulletin romand contre l'armement atomique, décembre 1967; "Le jeu de la guerre", Tribune de Genève, 10 avril 1967.

32 Voir Yvonne Baby, "La bombe", Le Monde, 3 septembre 1966.

33 Jean-Pierre Moulin, "La lettre de Paris. War Game", Tribune de Lausanne, 12 avril 1967.

34 Voir Claude Vallon, "La bombe de Peter Watkins", Feuille d'avis de Lausanne, 16 septembre 1967; Freddy Buache, "La bombe", Tribune de Lausanne, 17 septembre 1967.

35 Voir Louis Marcorelles, "The War Game. Un film de Peter Watkins", Gazette de Lausanne, 16 avril 1967.

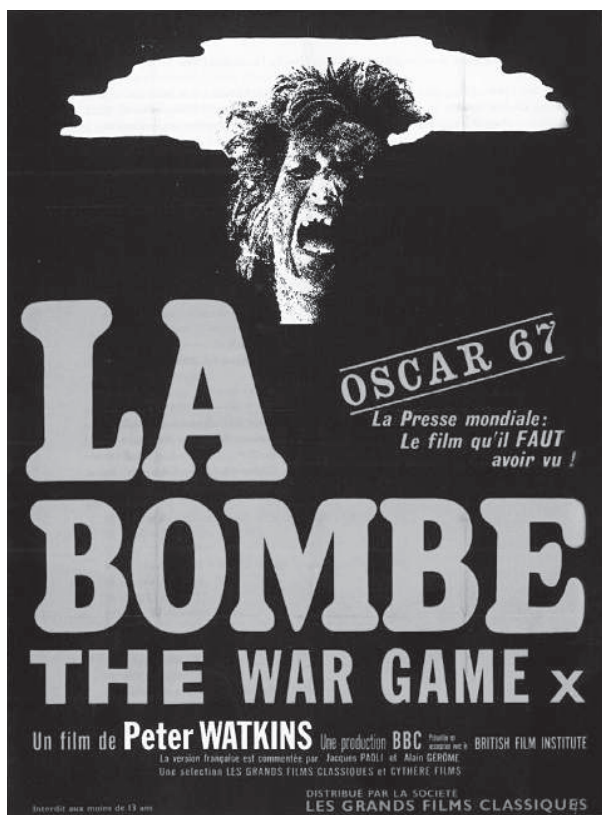

reposant sur la citation ou la transposition de codes audio-visuels dont la structure morphologique demeure reconnaissable) et comme une "performance» en acte (impliquant un processus de confusion entre la personne et le rôle mis en scène). Watkins conçoit explicitement ses films sur un mode paradoxal, notoirement dans The War Game, en mettant en scène les effets de l'explosion de bombes nucléaires «à la manière d'une reconstruction réaliste-documentaire $\mathbf{2 7}$ (il peut également présenter son film comme un «documentaire imaginaire» 28). Problématisant toute distinction entre "document» et "fiction", il se place d'emblée dans le domaine de la virtualité - position qui retient l'attention des journalistes, qui ne manquent pas d'exploiter l'ambivalence constitutive de ce genre dans leurs critiques. Dans les coupures de presse recueillies à la Cinémathèque Suisse ${ }^{29}$, par exemple, on trouve de nombreuses variations autour de la formule oxymorique "réaliste-documentaire" pour rendre compte de The War Game, à l'instar des mots composés "réalité-fiction » $\mathbf{3 0}$, «documentaire-fiction» $\mathbf{3 1}$, «documentaire-fictif» $\mathbf{3 2}$, «document-fiction» $\mathbf{3 3}$, «documentaire d'anticipation» $\mathbf{3 4}$, "reportage d'anticipation» $\mathbf{3 5}$, «actualités reconstituées» $\mathbf{3 6}$, «documentaire préventif d'anticipation» $\mathbf{3 7}$, ou encore "Actualités de l'avenir» $\mathbf{3 8}$. Comme le synthétise un article paru dans la Libre Belgique, ce "film d'anticipation", présenté comme un «document inventé» ou du "réalisme au 
second degré", "se sert d'une affabulation pour imiter certains procédés de reproduction et d'interprétation d'événements authentiques particuliers à l'image filmique» (se signalant "par un caractère de 〈vérité brute > inhérent aux bandes d'actualités et aussi par une impression de réalité immédiate donnée par les émissions de télévision 〈en direct〉») 39. On pourrait également rapporter les propos, quelque peu lyriques, de Jean-Louis Bory qui compare The War Game (ici renommé En Angleterre bombardée) à la stratégie de Kevin Brownlow et d'Andrew Mollo dans It Happened Here (En Angleterre occupée, 196540), mettant en scène l'invasion de la Grande-Bretagne par les nazis en 1940 :

"On part d'une hypothèse plausible, mais «En Angleterre occupée` relevait de la rétrospection - it happened here; ‘ En Angleterre bombardée> relève de l'anticipation - it could happen here, it will happen here. [...] En fait, tout est présent, de ce présent indiscutable de l'évidence cinématographique. Puissance qu'aucun article, fût-il de Sartre, aucun sermon, fût-il de Bertrand Russell, n'atteindra jamais. „41

Dans tous les cas, la notion de "politique-fiction", qui se systématise dans la presse francophone à partir de 1969 en regard de The Gladiators $\mathbf{4 2}$, ressaisit bien les enjeux de la démarche de Watkins: la "reconstruction réaliste-documentaire», que celle-ci porte sur le passé ou un futur proche, est au service d'un projet d'agitation politique ou de revendication sociale (d'où le recours à la catégorie parallèle de «sociétéfiction» $\mathbf{4 3}$ dans d'autres critiques). Il faut néanmoins distinguer le projet spécifique de Watkins de la catégorie lâche et sous-déterminée de "politique-fiction» qui désigne dans les années 1960 un sous-ensemble

The War Game (La bombe, 1965)

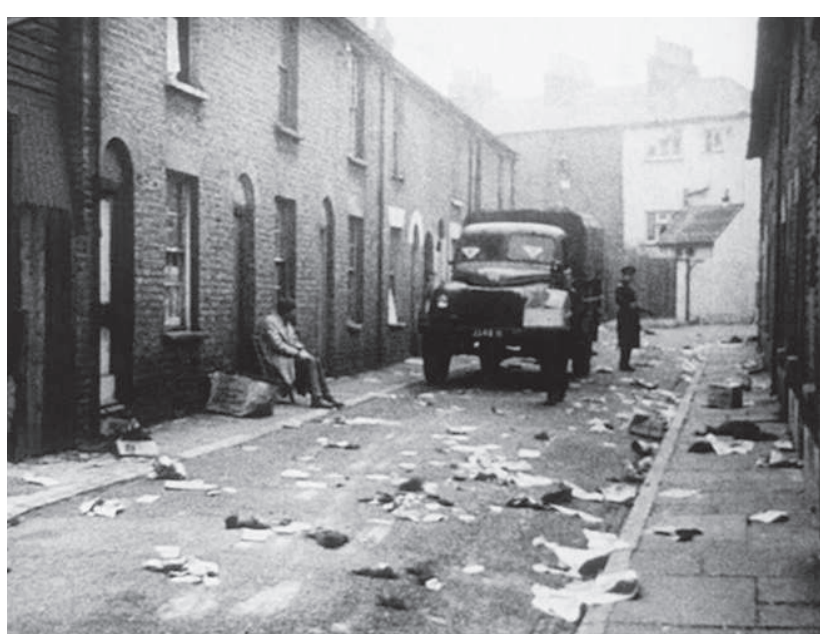

36 Voir "La bombe", Journal de Genève, 10 avril 1967; François Rochat, "La bombe de Peter Watkins ", Gazette de Lausanne, 16 avril 1967; "Le jeu de la guerre ou l'atroce réalité de demain ", Voix ouvrière, 3 juin 1967.

37 Voir Stefan Crozet, "The War Game, documentaire préventif d'anticipation", Jeune cinéma, n 17 , octobre 1966.

38 Voir "Pierre Billard a vu: La bombe", L'Express, 3 avril 1967.

39 "Le réalisme cosmique de Peter Watkins: La bombe", Libre Belgique, 30 novembre 1967.

40 Peter Watkins a participé à ce film amateur qui a, en fin de compte, bénéficié de l'aide financière de Tony Richardson, comme le précise Kevin Brownlow lui-même (How It Happened Here: the Making of a Film, New York, Doubleday, 1968, cité par James M. Welsh, Peter Watkins, op. cit., pp. 120-121).

41 Jean-Louis Bory, "En forme de cri", Nouvel Observateur, 5 avril 1967. La référence à Sartre et à Russell renvoie au Tribunal international de crimes de guerre, fondé en novembre 1966 dans le contexte de l'opposition des intellectuels de gauche à la guerre du Vietnam.

42 Voir Jean de Baroncelli, "The Gladiators", Le Monde, 11 novembre 1969; Jean-Louis Bory, "La guerre à bon marché", Le Nouvel observateur, 17 novembre 1969; B. C., "The Gladiators ", Positif, $\mathrm{n}^{\circ} 110$, novembre 1969, p. 52; J. P. S., "Les gladiateurs", Revue du cinéma - Image et son, $\mathrm{n}^{\circ} 236$, février 1970 , p. 138. Robert Chazal va jusqu'à proposer le nom composé "film politique-macaroni " pour rendre compte de Gladiateurs (Robert Chazal, "Les gladiateurs: drôle de guerre", France-Soir, 18 novembre 1969).

43 Raymond Durgnat théorise la catégorie de "society-fiction" par rapport à The War Game, Privilege, The Gladiators et Punishment Park (Raymond Durgnat, "The Great British Phantasmagoria ", Film Comment, vol. 13, n ${ }^{\circ} 3$, maijuin 1977, pp. 48-53). En 1967, George Sadoul envisageait déjà Privilege à travers la notion de "social-fiction" (Georges Sadoul, "Des stars aux idoles", Les lettres françaises, 28 juin 1967). 
44 Dans un numéro spécial de l'organe cinéphilique français Cinéma consacré aux Etats-Unis, la "politique-fiction" apparaît ainsi comme un cinéma de "genre" typiquement américain, au même titre que la comédie, le western, le film noir, le musical, le film historique, le fantastique et le film de guerre, mais aussi de pratiques moins codifiées telles que l'animation ou l'underground. Sous la plume de Roland Lacourbe, qui mentionne The War Game de Peter Watkins, la "politique-fiction" renvoie à des films d'anticipation populaires reflétant la peur d'une guerre atomique depuis les années 1940. (Voir Roland Lacourbe, "La politique-fiction", Cinéma 69, n 132, janvier 1969, pp. 61-64.) Dans un registre plus scientifique, le terme de "politique-fiction" ou, plus généralement, de "fictions d'anticipation politique" est mobilisé dans les études littéraires: voir notamment Michel Prat, Alain Sebbah (éd.), "Fictions d'anticipation politique", Eidôlon, n 73 , novembre 2006, Presses universitaires de Bordeaux.

45 Nous pensons à The Edge (1968), Milestone (1969) et surtout Ice (1975) de Robert Kramer, qui projettent dans un futur proche les revendications et les contradictions internes de communautés d'activistes, qui ont parfois recours à la violence armée. de la littérature et du cinéma de science-fiction ${ }^{\mathbf{4 4}}$, mobilisant un imaginaire dystopique, notamment la menace de la bombe A. Contrairement au cinéma du "samedi soir", qui exploite à travers des conventions de genre bien éprouvées des pulsions anxiogènes ancrées dans l'imaginaire collectif et liées au contexte de la guerre froide, les films de Watkins reposent sur un minutieux travail de recherche, pour éprouver sur le plan symbolique un scénario ou une hypothèse politique, à l'instar de certains films de Robert Kramer45. En mettant en scène un univers imaginaire ou reconstitué à travers les marqueurs stylistiques d'un document pris sur le vif, Watkins récuse toute prétention à la présentation objective de faits donnés comme authentiques ou véridiques. Mobiliser, comme le font régulièrement les journalistes, la catégorie de "cinémavérité» ou de "cinéma direct» (en faisant par ailleurs l'économie de toute définition ou recensement historique de ces pratiques) pour désigner ses reportages de "politique-fiction", ne permet pas de rendre compte de la mise en jeu consciente de conventions génériques hétérogènes, instaurant un pacte de lecture pour le moins équivoque avec le spectateur. L'intention première de Watkins est de saper ou déconstruire la distinction entre le mode documentaire et le mode fictionnel, afin de souli-
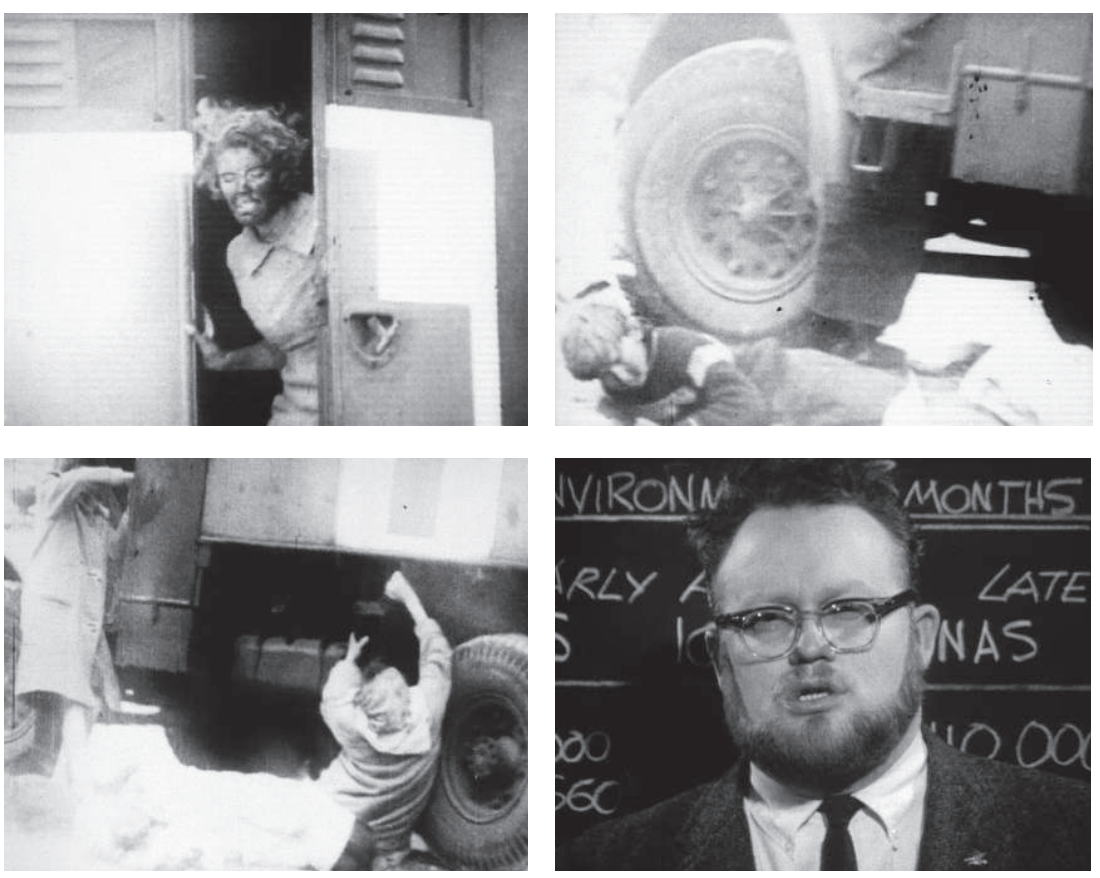
gner le caractère codifié de toute représentation, fût-elle présentée avec l'autorité des faits.

The War Game condense les enjeux du dispositif de "politiquefiction" imaginé par Watkins. La BBC a refusé de diffuser ce reportage d'agitation politique ${ }^{46}$, à cause du caractère horrifique et traumatique de la représentation d'une guerre nucléaire à l'écran. En un sens, Watkins proposait de transposer dans la sphère télévisuelle le geste d'Orson Welles qui donnait à entendre, en 1938, La Guerre des mondes 47 à travers le format d'un reportage radiophonique. Watkins exacerbe les procédés d'authentification des événements qui caractérisent les actualités télévisées, dans le but d'impliquer émotionnellement le spectateur, mais aussi de dénaturaliser ces conventions. L'explosion de la bombe A se traduit à travers une série d'images-chocs, dont l'artificialité de la mise en scène est soulignée, mais qui n'en sollicite pas moins les affects du spectateur. Cette fable politique recourt aux artifices rhétoriques de la feintise ou du simulacre, tout en suscitant la participation imaginaire du spectateur, qui se projette dans la situation mise en scène (la publication du film sous la forme d'un livre illustré en 196748 rend manifeste sa relation à la forme littéraire de la «feintise»). Dans The War Game, la
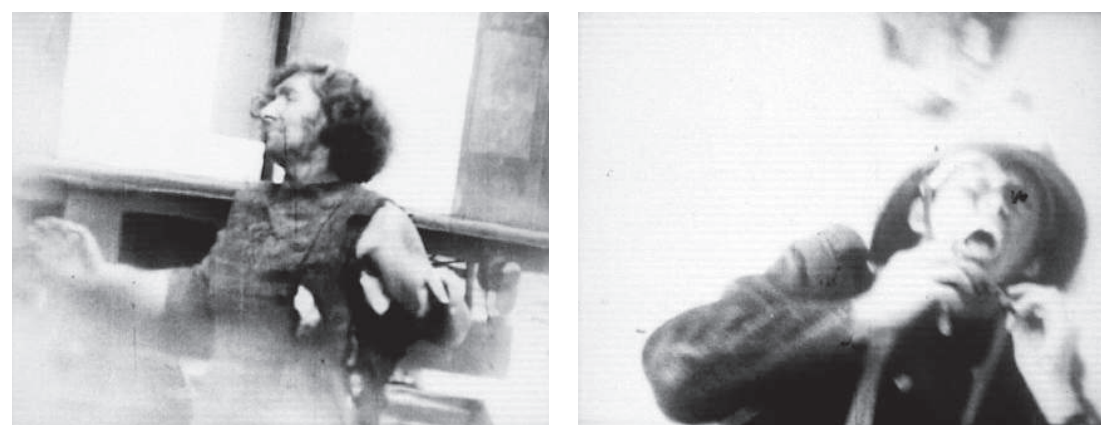

In the present version of the Civil

Defence pamphlet listing the various

items to be taken into a refuge-room

for life in a post- nuclear-attack

Britain, are the following words:

"... and a box containing birth and

marriage certificates, savings bank

books and National Health Medical Cards".
46 The War Game devait être diffusé le 6 août 1965 , pour commémorer le vingtième "anniversaire" d'Hiroshima. La BBC multiplie les atermoiements, avant de censurer le film pendant vingt ans.

47 La Guerre des mondes est un récit de science-fiction de H. G. Wells publié en 1898. Le parallèle a déjà été noté par un journaliste de La Suisse: "[...] j'imagine volontiers que le scénario, conçu comme un documentaire à l'instar de l'adaptation radiophonique par Orson Welles de La Guerre des mondes, en 1938, aurait provoqué un affolement considérable en GrandeBretagne." "La bombe", La Suisse, 18 septembre 1967).

48 Voir Peter Watkins, The War Game, New York, Avon Books, 1967. La page de titre à l'intérieur du livre annonce: "La Troisième guerre mondiale. Cela pourrait commencer ainsi. C'est LA GUERRE MONDIALE. ". 
49 Notons à cet égard que l'Office fédéral de la protection civile fait circuler en Suisse romande (en tout cas à Neuchâtel et Delémont) une missive pour marquer l'intérêt du film de Watkins, tout en rassurant le citoyen: tout est ici prévu pour faire face à une attaque nucléaire (voir "The War Game - La Bombe. Un enfer terrestre... sans protection civile véritablement organisée ", 1967)...

50 Dans les encarts de la presse romande annonçant la sortie de The War Game, le film ("Absolument sensationnel!") est "déconseillé aux personnes nerveuses et impressionnables" (Feuille d'avis de Lausanne, 10 mai 1967). Notons encore que film est couronné en 1967 par l'Oscar du meilleur documentaire. critique porte tant sur le plan de l'énoncé (en dénonçant l'insuffisance des mesures prises en cas de conflit nucléaire et la désinformation du public 49) que sur celui de l'énonciation (à travers la mise en scène spectaculaire d'un événement qui échappe à toute représentation, induisant un effet de choc 50). L'amorce blanche intercalée dans le film pour signifier l'impact de la bombe cristallise ces deux aspects; elle se prolonge ou se répercute à travers une série d'images spectaculaires mettant en scène les poses outrancières des acteurs et la mise à sac des décors, le montage permettant de visualiser un chaos excédant toute figuration. La voix over et la voix off, incarnées par deux présentateurs de la BBC (Dick Graham et Michael Aspel), décrivent les faits et prennent la mesure de l'étendue des dégâts à travers une perspective factualiste, parfois clinique. The War Game alterne entre des entretiens filmés ou des déclarations de personnalités publiques, des flashs d'information et des scènes de destruction (l'explosion de missiles thermonucléaires sur la Grande-Bretagne, générant notamment une tempête de feu qui constitue l'un des morceaux de bravoure du film).

Une scène est exemplaire à cet égard: une bombe explose à Chattham, avant d'atteindre sa cible; pour représenter son impact, l'écran est envahi par une fumée blanche qui trouble la lisibilité de la représentation. La voix over décrit sans empathie les symptômes subis par les victimes, tandis que cinq plans se succèdent à l'écran, la prédominance du blanc amenuisant la démarcation des coupes franches:

«Lorsque la teneur en dioxyde de carbone de l'air inhalé dépasse $30 \%$, cela entraîne: diminution de la capacité respiratoire, baisse de la tension artérielle, coma, perte de réflexes et anesthésie. Lorsque la teneur en monoxyde de carbone de l'air dépasse 1,28\%, la mort survient dans les 3 minutes. C'est ça, la guerre nucléaire».

Les corps, en proie à l'asphyxie, suivent un mouvement inéluctable de chute, tendant à se confondre avec la poussière des gravats, à travers une esthétique expressionniste, qui rompt avec les codes du reportage de guerre. Une femme s'extrait péniblement hors d'un camion de la Croix-Rouge, cadré en plan rapproché; la porte du camion se referme, la fumée envahit l'écran, et la femme, qui se révèle être une infirmière, s'effondre au pied du camion. Par un effet de fondu enchaîné induit par la fumée (malgré une coupe sèche), apparaissent un enfant, puis une autre infirmière, qui se dirigent vers le corps inanimé de la jeune femme, étendu auprès du châssis et des roues du camion; dans la continuité du plan toujours, les trois personnages gisent au sol. Suite à une coupe franche, une autre infirmière sort à son tour du camion, et tombe également. Autre plan, un pompier s'approche du véhicule de la Croix-Rouge, 

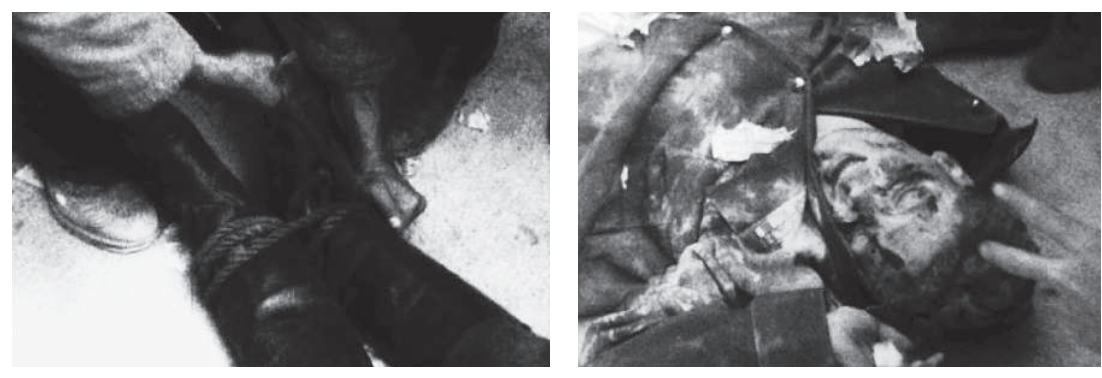

avant de s'affaler. Enfin, dernier plan de cette scène, une vieille dame s'effondre au pied du camion. Cette séquence saisissante est intercalée entre une interview avec un stratège nucléaire américain et l'exposé des consignes à suivre dans les abris extrait d'une brochure éditée par la défense civile. La démarche de Watkins évoque ici les films soviétiques d'agit prop: dramatisation des conditions d'hygiène et de vie, jeu behavioriste des acteurs non professionnels, procédés de montage discursif. Tout au moins Watkins conjoint-il ici avec efficacité l'objectivité sociale des productions de la télévision britannique et le caractère expérimental du cinéma amateur, qui constituent ses deux champs de référence immédiats.

Rappelons en effet que Watkins a tout d'abord éprouvé le dispositif énonciatif de la "politique-fiction» à travers les courts métrages qu'il a tournés en $8 \mathrm{~mm}$ ou en $16 \mathrm{~mm}$ avec la troupe amateur Playcraft Players. Le style indistinctement "objectivant» et "expressionniste» de Forgotten Faces (1960) tire selon nous les leçons du principe de la reconstitution, tel qu'il s'appliquait dans certains documentaires militants des années 1930 - nous pensons à des films aussi différents que Borinage (Storck et Ivens, 1933) ou Las Hurdes (Buñuel, 1932). Cette reconstitution du soulèvement de Budapest repose sur le recours à de la documentation iconographique (des photographies parues dans Times et Paris-Match), et implique une transfiguration du milieu filmé, les acteurs se projetant dans la Hongrie de 1956 51. Dans ce film tourné en $16 \mathrm{~mm}$, Watkins met en jeu un dispositif performatif qui évoque par certains aspects un happening pour la caméra (comme on dit: une performance d'atelier, destinée à la captation par la caméra ou la vidéo): exemplairement, lors de l'assaut d'une ambulance par la foule, qui occasionne l'exécution publique de membres de la police secrète, les prises de vue alternant entre des gros plans sur les visages effarés ou les jambes entravées des pandores, et des vues d'ensemble de mouvements de foule, pour finir sur un plan d'un policier pendu, qui évoque les

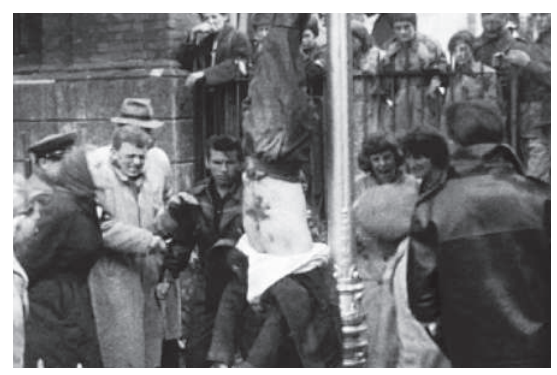

Forgotten Faces (1960) $\mathbf{5 1}$ Voir Philippe Durand, "Le monde du cinéma amateur", Cinéma 63, n 80, novembre 1963, pp. 123-125; Sébastien Layerle, "Une juste appropriation des faits. The Forgotten Faces et les cannées Playcraft, (1956-1962) ", op. cit. 
photographies de suppliciés reproduites par Georges Bataille dans la revue Documents.

\section{Performance et performativité}

52 Voir notamment Richard Schechner, Essays on Performance Theory, 1970-1976, New York, Drama Book Specialists, 1977 (rappelons que Schechner est le fondateur de The Performance Group en 1967, puis de The Wooster Group en 1980, et l'éditeur de The Tulane Drama Review). Watkins transpose dans le domaine filmique les pratiques du théâtre politique qui reposent sur l'improvisation des acteurs et sur l'appréhension de l'espace scénique comme le lieu d'une performance, sa démarche étant assimilable à la logique des happenings et des environnements qui se multiplient dans les années 1960 (voir les trois recueils de référence suivants: Michael Kirby, Happenings, New York, E. P. Dutton, 1965; Richard Kostelanetz, The Theater of Mixed Means, New York, Dial Press, 1966; Allan Kaprow, Assemblage, Environments and Happenings, New York, Abrams, 1966).

La théorie de la performance $\mathbf{5 2}$ permet selon nous d'expliciter les principaux enjeux de la démarche cinématographique de Watkins, tout en maintenant une distinction entre la mise en scène et la mise en cadre, la direction d'acteurs et la composition de l'image. Le travail collectif avec les acteurs, la confusion entre la personne et le personnage, l'indistinction entre la mise en scène et la situation reconstituée ou anticipée, reposent sur un dispositif performatif sciemment activé par Watkins, mais qui rapidement s'autonomise et excède toute possibilité de maîtrise de la part du metteur en scène. L'opérateur et le preneur de son, dirigés par Watkins, se trouvent alors dans la situation d'un cinéaste qui capte une performance qui lui échappe, ne pouvant dès lors qu'en extraire des fragments, en exposer des bribes. La phase de la recherche historique et de la documentation est renégociée lors des répétitions et du tournage: c'est grâce à l'implication des acteurs amateurs dans la construction de leur personnage que les gestes peuvent se libérer, la parole se déployer. Le «scénario" imaginé par Watkins est revécu, performé lors du tournage. Une scène emblématique de Punishment Park, qui prend pour point de départ la loi de
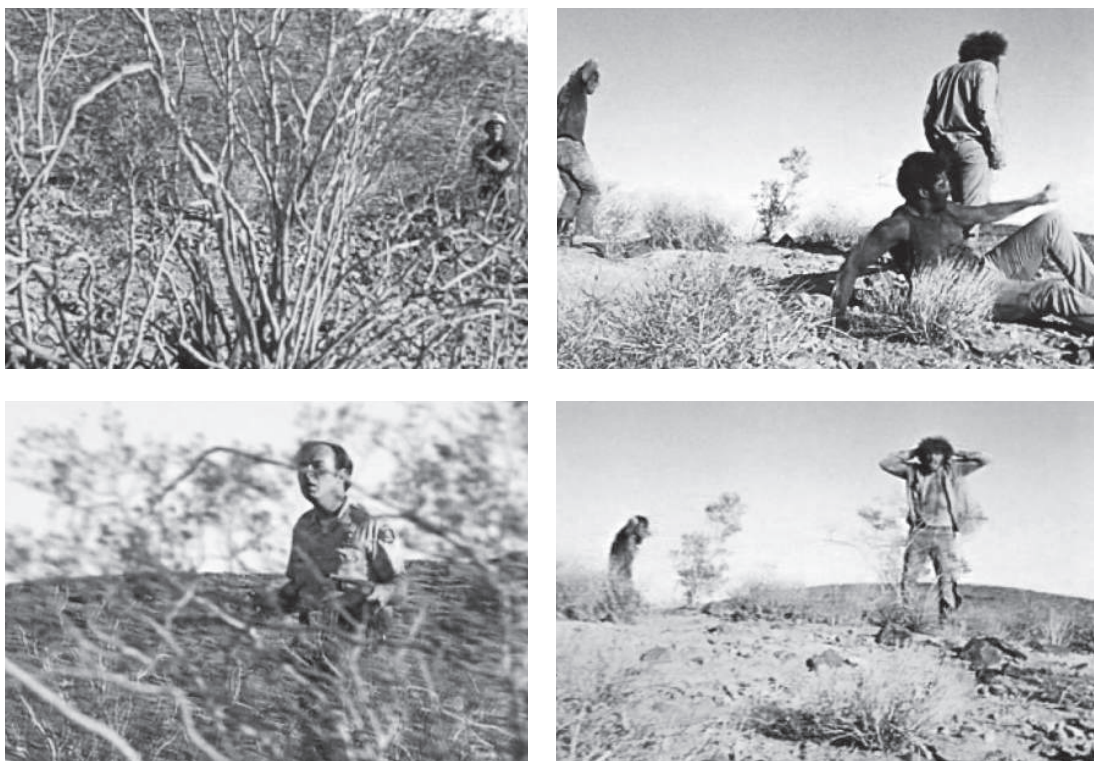
McCarran de 1950, restaurée par Nixon en février 1970, permet d'en prendre la mesure.

Punishment Park, qui alterne rapidement entre les séquences de jugement hâtif d'opposants au régime américain (à travers une référence explicite au procès des Huit de Chicago) et des scènes de poursuite dans l'espace asphyxiant du désert du Parc de répression, mobilise une structure duelle, instituant une opposition entre voyant/vu, qui n'exclut pas pour autant une possibilité de réversibilité : le regard est médiatisé par la lunette du fusil mais aussi par la caméra d'une équipe de télévision de la BBC qui couvre l'événement, point de vue relayé par l'opératrice du film de Watkins. Les mouvements de la caméra, multipliant les zooms et la mise au point progressive, le montage rapide d'images tournées en plans-séquences, miment les conditions de tournage d'un reportage de guerre, la voix over et la poursuite des dialogues par-delà l'unité du plan contribuant à linéariser les séquences (l'introduction subreptice d'images semi-subjectives pour signifier l'épuisement rompant avec ce régime de représentation). Les journalistes, témoins et garde-fous de cette chasse à l'homme, assistent au déséquilibre entre un arsenal répressif (juridique d'abord, policier ensuite) et un groupe rapidement dispersé et réprimé d'opposants à la guerre du Vietnam, de militants des Black Panthers, de pacifistes, d'étudiants, d'artistes et d'intellectuels de gauche. La caméra, qui mime d'abord la position de témoin impartial, multiplie les angles de focalisation: elle s'entretient avec les prisonniers comme avec les gardiens, restitue le point de vue des instances de la répression et accompagne les victimes dans leur fuite. Progressivement, elle s'implique de plus en plus dans les événements mis en scène.

Dans le climax du film, une indistinction s'opère entre les points de vue de l'équipe de télévision, de l'opératrice de Punishment Park, de l'instance d'énonciation filmique et du réalisateur lui-même. La caméra se déplace de l'espace des gardiens à celui des prisonniers, revient vers le camp des représentants de l'ordre (l'un d'eux s'exclamant: "Sale nègre!»), guette un gardien à l'affût derrière un bosquet (l'on entend alors l'équipe TV qui affirme sa présence pour désamorcer la situation) qui s'avance deux armes à la main, pour revenir finalement sur les militants les bras levés («Filmez, ils vont nous tuer!»). Deux plans sont alors focalisés sur les prisonniers, le premier cadrant un cadavre, le second les militants qui s'entretiennent avec un journaliste, tandis que le lien entre les deux est assuré par un commentaire en voix off ( $O \mathrm{On}$ va s'en tirer»). L'action reprend en un plan plus large, pour immédiatement zoomer sur les militants encerclés par un gardien et un policier (en voix over:
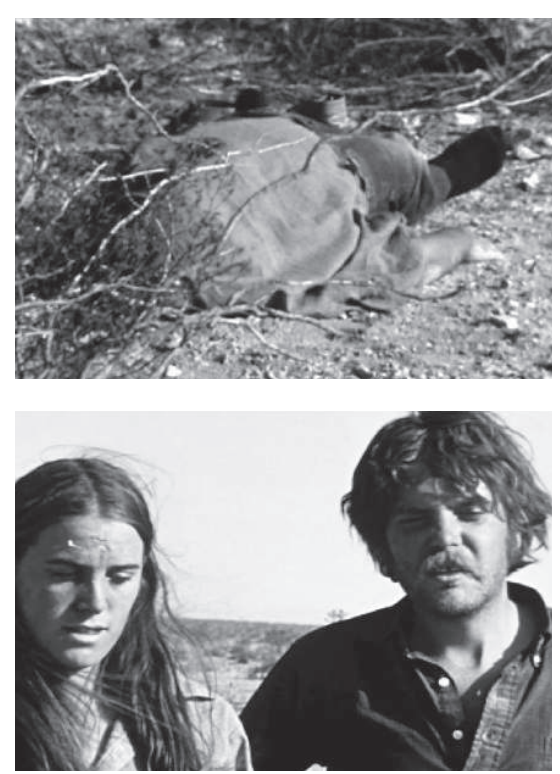
Punishment Park (1970)
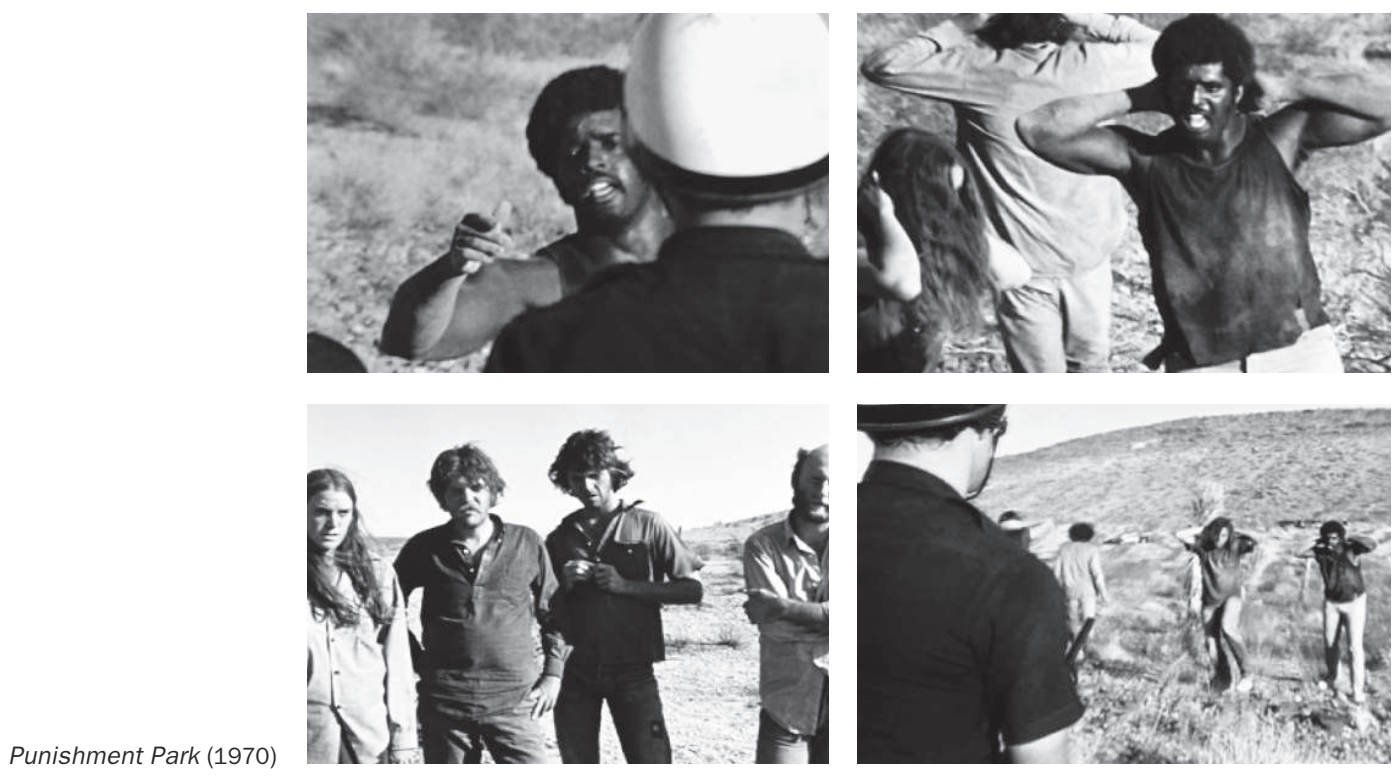

53 Watkins, en voix off, incarne dans l'univers diégétique du film un journaliste de la BBC. A l'occasion du tournage de cette scène, il confond mise en scène et événement afilmique, pensant à tort qu'un soldat a ouvert le feu à balles réelles sur deux manifestants; en réalité, ceux-ci ne font que simuler leur propre mort (voir Joseph A. Gomez, Peter Watkins, op. cit., p. 105).

«L'alternative est de réagir à cette autodestruction passivement ou violemment»); le bras du policier en amorce fait subrepticement écran à la scène, mais la caméra recadre aussitôt le policier qui tient en respect les militants. Suite à une coupe franche, un plan rapproché montre les prisonniers en situation d'entretien avec les journalistes. Une opposition est alors construite entre le premier plan, occupé par les forces de l'ordre, et l'arrière-plan, où se trouvent les prisonniers; mais à la faveur d'un zoom avant, la scène est recadrée sur les militants qui jettent des pierres contre des soldats à l'arrière-plan, eux-mêmes ne tardant pas à faire face à la caméra, au gré du zoom avant qui se poursuit. Un plan de coupe intervient, un prisonnier répondant aux questions de l'équipe de la BBC. Suivant la dynamique du champ/contrechamp, ce face-à-face est repris et inversé : les soldats ouvrent le feu en direction de la caméra; l'opératrice amorce un zoom arrière, décadre et recadre frénétiquement l'espace, avant de reprendre dans le champ les soldats qui tirent et les militants tombés à terre, bientôt rejoints par un policier, tandis que l'on entend, au milieu des cris des acteurs, l'injonction de Watkins: «Mon Dieu! Coupez! Coupez!»53. Cette séquence se clôt sur un drapeau américain, cadré en gros plan, qui fait écho à la première séquence du film et au but inatteignable qui a été assigné aux prisonniers. L'on assiste dans cette scène de climax à une confusion littérale entre mise en scène fictionnelle et événement pris sur le vif: le dispositif 

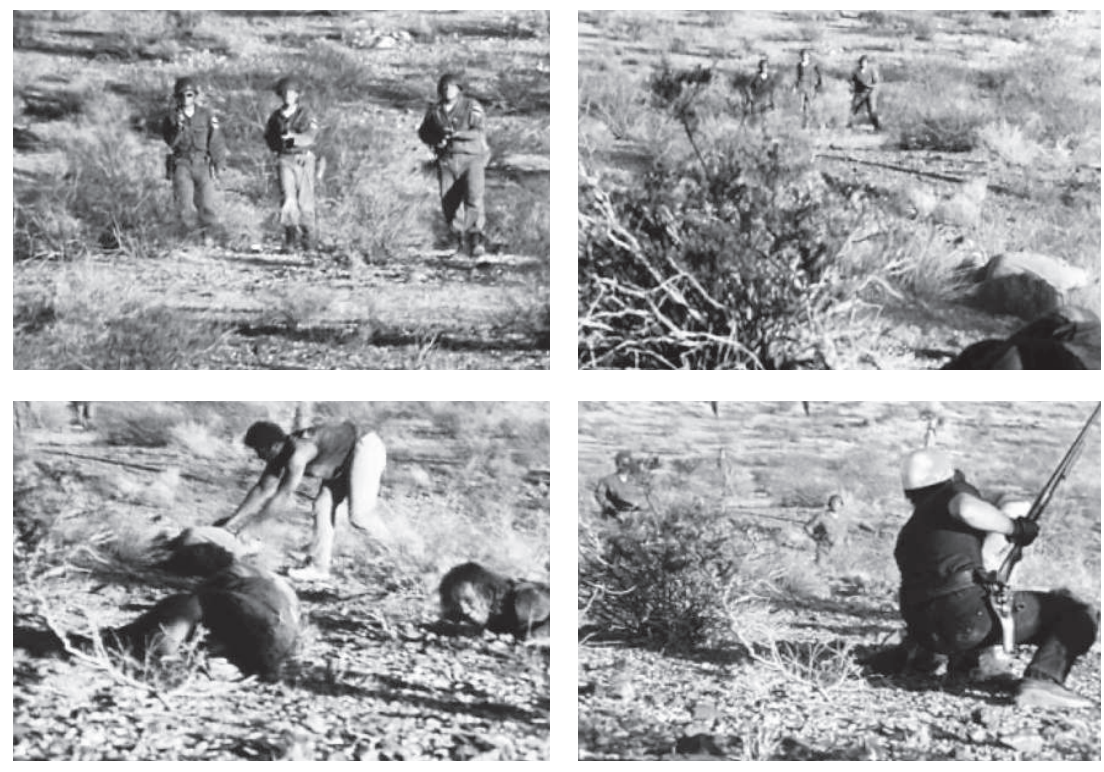

performatif ${ }^{54}$ que Watkins a mis en place s'est autonomisé, le réalisateur perdant le contrôle du tournage. Punishment Park ne se laisse ainsi pas réduire à la logique paranoïaque de son scénario : le dispositif censément régulé du parc punitif est traversé par des moments de perte de maitrise, de la part des acteurs et de l'équipe de tournage.

Ce dispositif performatif correspond à un happening pour la caméra, dans le sens où Allan Kaprow et, à sa suite, Richard Schechner comprennent ce terme. Dans l'anthologie Assemblage, Environments and Happenings, Kaprow établit sept axiomes définitoires du happening qui pourraient être appliqués au dispositif de tournage que Watkins instaure dans Punishment Park:

«(A) Il faut que la limite entre l'art et la vie soit aussi poreuse, voire indiscernable, que possible. [...] Il se passe toujours quelque chose à ce point de rencontre [...]. [...]

(C) Il faut que la représentation d'un Happening se déroule dans plusieurs lieux très vastes, et qu'on en change de temps en temps. [...]

(D) Il faut que la durée, qui suit de près les considérations valables pour l'espace, soit variable et discontinue. [...] Par-dessus tout, il est question de durée 〈réelle〉 ou ‘vécue , à distinguer de la durée conceptuelle. [...] (E) Il ne faut pas représenter un Happening plus d'une fois. [...]

(F) Par conséquent, il faut complètement bannir le public. Tous les éléments - les personnes, l'espace, les matériaux et le caractère particuliers
54 En 1973, Watkins peut ainsi écrire: "[...] Punishment Park s'inscrit précisément dans la voie d'expérience inaugurée par The War Game et Culloden - à savoir susciter une implication auprès du public de sorte à ce qu'il [...] se sente contraint à agir autrement." (Peter Watkins, "Punishment Park and Dissent in the West", op. cit., p. 296). 
55 Allan Kaprow, Assemblage, Environments and Happenings, op. cit., pp. 188-194, cité par Richard Schechner, "Participation", Performance. Expérimentation et théorie du théâtre aux USA (Christian Biet, Anne Cuisset et Marie Pecorari, éd.), Montreuil, Editions Théâtrales, 2008, p. 213 [R. Schechner, Environmental Theater, New York, Hawthorn Books, 1973, p. 61].

56 Voir notamment Allan Kaprow, 18 Happenings in 6 Parts, Göttingen, Steidl Hauser \& Wirth, 2007.

57 Richard Schechner, "Participation", op. cit. p. 223. Schechner cite le Petit organon pour le théâtre de Brecht: "Exiger du comédien qu'il se tienne sur le plateau sous une double apparence, à la fois comme Laughton et comme Galilée, exiger de Laughton le montreur qu'il ne disparaisse pas derrière Galilée le montré [...], c'est simplement se refuser à dissimuler plus longtemps le processus réel et profane qui se déroule sur la scène. Car c'est tout de même Laughton que l'on trouve sur le plateau, Laughton qui nous montre comment il se représente Galilée! " (Bertolt Brecht, Petit organon pour le théâtre, Paris, L'Arche, 1970, p. 65 ["Kleines Organon für das Theater", Sinn und Form (Johannes R. Becher, Paul Wiegler, éd., "Sonderheft Bertolt Brecht"), Berlin, Verlag Rütten und Loening, 1949, pp. 11-40]). à l'environnement, la durée - sont ainsi à même d'être intégrés au Happening. [...]

(G) La composition d'un Happening se développe exactement comme celle d'un Assemblage ou d'un Environnement, en d'autres termes, celui-ci se déroule comme un collage d'événements de durée limitée et situés dans des lieux définis. $[. .]. » \mathbf{5 5}$

Nous sommes conscients du forçage interprétatif que nous opérons vis-à-vis du manifeste de Kaprow, qui décrit une pratique performative qui ne correspond aucunement à une situation de tournage. Mais mobilisés en tant que relais analogique, les axiomes de Kaprow éclairent en retour la logique des reportages de "politique-fiction" de Watkins. L'indistinction entre l'art et la vie, ou plutôt ici la représentation et le réel, la vastitude des lieux et la discontinuité de la durée vécue, le caractère unique et non répétable de l'action et de la prise de vue, l'intégration performative des acteurs, des personnages et des journalistes mis en scène dans l'espace du tournage, et jusqu'à la forme du collage ou de l'assemblage, sont bien mis en jeu dans Punishment Park. Mais à la différence des happenings de Kaprow 56, la performance ou l'événement se déroulent littéralement sans public, leur captation par la caméra portée à l'épaule contribuant à fragmenter l'action (qui peut en effet être décrite comme «un collage d'événements de durée limitée» - le temps d'un chargeur - "situés dans des lieux définis»- les tentes et le désert de Punishment Park). La confusion entre la représentation et le réel, la personne et le personnage, comme le montre bien Schechner, n'est pas étrangère à «l'effet V» (VerfremdungEffekt) décrit par Brecht, qui "n'a pas pour but de bannir 〈l'émotion" du théâtre, mais de mettre en avant le rôle double de l'interprète, sa fonction difficile dans l'espace transitoire qu'est le théâtre» - «cette illusion" qui "peut être mise au service de la désillusion (c'est-à-dire du dévoilement)» $\mathbf{5 7}$.

Les reportages de "politique-fiction" et les reconstitutions historiques de Watkins participent à la logique du "théâtre environnemental» théorisé par Schechner, qui constitue une variante autour de la structure du happening. Celui-ci soutient que les manifestations de rue et leur scène politique fortement théâtralisée constituent le modèle du théâtre performatif:

«Une marche pour les libertés civiques ou contre la guerre du Viêt Nam est une performance: les rues deviennent la scène et l'on joue pour des spectateurs à la fois présents et pour ceux qui regardent la télévision de chez eux ou lisent le journal. [...] Cette utilisation croissante de l'espace public pour des activités de plein air, répétées, 
allant des manifestations aux artistes de rue, commence à peser sur

le théâtre en salle.» $\mathbf{5 8}$

Le cinéma, à la différence du "théâtre en salle", s’inscrit directement dans l'espace public; et Watkins n'a de cesse de créer des situations qui sont de l'ordre de la performance et de la manifestation, sans aller pour autant jusqu'au «théâtre de guérilla». Regrettant l'absence de prise en compte des "enjeux esthétiques» des marches et des manifestations de protestation, Schechner analyse leur "utilisation créative» de l'environnement, les rues se transformant en "espaces publics", "terrains d'essais» et "théâtres dans lesquels se jouaient des moralités»59. Revenant sur ses propres pièces d'intervention politique dans l'espace public, en l'occurrence la série d'événements intitulés Guerrilla Warfare et joués dans 33 lieux à New York le 28 octobre 1967, Schechner décrit l'implication des spectateurs en des termes qui évoquent la situation des acteurs des reportages de "politique-fiction» face à la caméra de Watkins :

"Une fois qu'une performance a 'pris forme> dans un espace [...], les spectateurs trouvent eux aussi leur place: il y a réciprocité du phénomène. Il arrive fréquemment que les spectateurs, en absence de placement fixe et d'informations sur la nature de la performance, se disposent d'une manière inattendue ; et, au cours de la performance, cette disposition change, ‘respire`, en suivant l'action, comme le font les interprètes. [...] Dans le théâtre environnemental, [...] les déplacements s'enchaînent $[. .$.$] comme dans un match de sport, où$ la progression des déplacements suit des règles et où c'est de la décision d'une personne ou d'un groupe que dépendent les réactions possibles. Les interprètes doivent savoir profiter de la mobilité du public et la considérer comme une partie flexible de l'environnement de la performance.» 60

L'analyse de Schechner permet de rendre compte des déplacements des groupes et des individus dans Punishment Park, où les coups sont imprévisibles et joués par des flux de mouvements plus que par une volonté individuelle, les lieux du tournage représentant une "partie flexible de l'environnement de la performance». Si nous élargissons ce dispositif à l'ensemble des films de "politique-fiction" et de reconstitution historique de Watkins, il est possible d'affirmer qu'à l'instar des happenings, l'événement peut «prendre forme» ou s'effilocher. Si le jeu est complètement maîtrisé, contrôlé, à l'instar de ce qui se passe dans The Gladiators, la performance n'a pas vraiment lieu, tout au plus est-elle représentée, simulée. Par contre, quand la situation performative s'autonomise, comme à la fin de Punishment Park ou dans de nombreuses séquences de La Commune, les réactions des participations deviennent inattendues
58 Richard Schechner, "Six axiomes pour le théâtre environnemental ", Performance. Expérimentation et théorie du théâtre aux USA, op. cit., pp. 131-132 ["Six Axioms for Environmental Theater", Environmental Theater, New and Expanded Edition, New York, Hawthorn, 1973]. Les six axiomes du théâtre environnemental, qui font directement écho aux sept thèses de Kaprow sur le happening, sont les suivants: "L'événement théâtral est un ensemble de transactions connexes"; "L'intégralité de l'espace est utilisé pour la performance"; "L'événement théâtral a lieu dans un espace totalement transformé ou dans un "espace trouvé" "; "L'attention est flexible et variable"; "Toutes les composantes du spectacle parlent leur propre langue"; "Le texte n'est pas forcément le point de départ ni le but du spectacle. Le texte verbal n'est pas indispensable».

59 /d., p. 137.

$60 / d .$, p. 139. 
61 "Il y a point de vue multiple quand plusieurs événements simultanés [...] sont répartis dans tout l'espace. [...] L'espace est organisé de manière à ce qu'aucun spectateur ne puisse tout voir. Les spectateurs sont contraints de se déplacer ou de réajuster leur regard, ou de choisir. [...] Recourir au point de vue multiple dans une performance signifie que celle-ci sera perçue différemment par chaque spectateur [...]." (/d., pp. 140-141).

62 "Dans le cas du point de vue localisé, les événements sont mis en scène de manière à ce qu'une partie seulement du public puisse les voir et les entendre. [...] Le point de vue localisé a l'avantage de rapprocher certaines scènes d'une partie du public de manière très directe. [...] La focalisation locale peut bien sûr être utilisée dans le cadre d'une focalisation multiple." (ld., pp. 141-142).

63 "Dire quelque chose provoquera souvent le plus souvent - certains effets sur les sentiments, les pensées, les actes de l'auditoire, ou de celui qui parle, ou d'autres personnes encore. Et l'on peut parler dans le dessein, l'intention, ou le propos de susciter ces effets. [...] Nous appellerons un tel acte un acte perlocutoire, ou une perlocution." (J. L. Austin, Quand dire, c'est faire, Paris, Seuil, 1970, p. 114 [How to Do Things with Words, Oxford, Oxford University Press, 1962]. Précisons que cet ouvrage recueille une série de conférences prononcées en 1955).

64 Richard Schechner, "Six axiomes pour le théâtre environnemental ", op. cit, p. 147. et font précisément événement. Il nous paraît indéniable que Punishment Park est construit selon un "point de vue multiple» $\mathbf{6 1}$, dans lequel s'enchâsse parfois un "point de vue localisé» 62, la flexibilité et la variabilité de la focalisation s'opposant à «l'unicité du point de vue» du théâtre ou du cinéma orthodoxes.

La notion d' "espace public oppositionnel» que nous avons appliquée au pôle de la réception et de la diffusion des films de Watkins, et que nous aurions pu élargir à leur production - tout au moins en ce qui concerne The Journey (1983-1986) - permet également de ressaisir leur mode de réalisation, reposant sur une forme ouverte. Car ce n'est pas seulement à un mouvement de décontextualisation de conventions formelles empruntées au reportage d'actualité ou de déterritorialisation de propos tenus par des représentants des instances officielles que l'on assiste dans les films de "politique-fiction" de Watkins, mais encore à la constitution d'un espace de parole pluriel et ouvert où les acteurs amateurs peuvent exprimer leur point de vue. S'il fallait mobiliser le modèle des actes de langage théorisé par John Austin, l'on pourrait soutenir que dans les films de Watkins les échanges de parole provoquent des effets et des actions chez les locuteurs et leurs destinataires, constituant ainsi des «actes perlocutoires" $\mathbf{6 3}$, dans un contexte de remise en cause de la définition des rôles sociaux. Car il s'agit encore pour Watkins de jeter le trouble dans la fixité des identités sociales et politiques, en vue de procéder à une remise en cause de leurs fonctions idéologiques. L'entreprise collective de La Commune, toute proportion gardée, pourrait être décrite dans les termes mêmes de Schechner qui commente son travail avec le New Orleans Group (NOG) à partir de la pièce Victimes du devoir de Ionesco:

«Dans le théâtre environnemental, la pièce n'est pas forcément première, il n'y a pas d'original, et tous ceux qui conçoivent le spectacle en sont les arbitres finaux. Cette < conception du spectacle> peut être le fait d'un seul auteur, d'un collectif ou d'une collaboration avec le public. Le NOG n'a pas ‘joué> la pièce de Ionesco, il a ‘joué avec la pièce`. Nous nous sommes confrontés à elle, nous avons creusé ses mots et ses thèmes, nous avons construit avec et à travers elle - et nous en avons retiré une vision personnelle. C'est là le cœur du théâtre environnemental.» 64

Certes, chez Watkins, le public n'est pas appelé à collaborer directement à la réalisation d'un film. Cependant, la «conception du spectacle» dans La Commune repose sur un travail collectif, où il s'agit de «jouer avec» les événements de la pièce ou, en l'occurrence, avec les enjeux de la guerre civile de 1871 en France, pour «creuser ses mots et ses 
thèmes». Plus largement, le cinéma de Watkins provoque la réaction du public: à un premier niveau, en sollicitant l'engagement politique et l'action civique des spectateurs, en réaction au choc traumatique suscité par la mise en scène de situations paroxystiques; à un deuxième niveau, en instituant un dispositif performatif dans lequel interagissent non hiérarchiquement les acteurs et l'équipe de tournage, invitant le public à constituer un espace communautaire alternatif; et à un troisième niveau, qui échappe à la maîtrise du vouloir-dire du réalisateur, en laissant libre cours à un happening excédant la scénarisation et l'improvisation des acteurs, le film constituant la trace d'une économie de la dépense (Watkins instaure les conditions de possibilité d'une ère d'échange appelant au potlatch, qui est actualisé par les acteurs). C'est la raison pour laquelle nous nous éloignons délibérément d'une lecture littérale du propos de Watkins - en interrogeant les points de rupture de son œuvre, nous soutenons que certains de ses films constituent une expérience authentiquement performative. 